\title{
SENSITIVE SEARCH FOR RADIO VARIABLES AND TRANSIENTS IN THE EXTENDED CHANDRA DEEP FIELD SOUTH
}

\author{
K. P. Mooley ${ }^{1}$, D. A. Frail ${ }^{2}$, E. O. OfeK ${ }^{3}$, N. A. Miller ${ }^{4}$, S. R. Kulkarni ${ }^{1}$, And A. Horesh ${ }^{1}$ \\ ${ }^{1}$ Cahill Center for Astronomy, MC 249-17, California Institute of Technology, Pasadena, CA 91125, USA \\ ${ }^{2}$ National Radio Astronomy Observatory, P.O. Box O, Socorro, NM 87801, USA \\ ${ }^{3}$ Benoziyo Center for Astrophysics, Faculty of Physics, The Weizmann Institute for Science, Rehovot 76100, Israel \\ ${ }^{4}$ Department of Astronomy, University of Maryland, College Park, MD 20742-2421, USA \\ Received 2012 December 16; accepted 2013 March 22; published 2013 April 25
}

\begin{abstract}
We report on an analysis of the Extended Chandra Deep Field South (E-CDFS) region using archival data from the Very Large Array, with the goal of studying radio variability and transients at the sub-milliJansky level. The 49 epochs of E-CDFS observations at $1.4 \mathrm{GHz}$ sample timescales from 1 day to 3 months. We find that only a fraction (1\%) of unresolved radio sources above $40 \mu \mathrm{Jy}$ are variable at the $4 \sigma$ level. There is no evidence that the fractional variability changes along with the known transition of radio-source populations below $1 \mathrm{mJy}$. Optical identifications of the sources show that the variable radio emission is associated with the central regions of an active galactic nucleus or a star-forming galaxy. After a detailed comparison of the efficacy of various source-finding algorithms, we use the best to carry out a transient search. No transients were found. This implies that the areal density of transients with peak flux density greater than $0.21 \mathrm{mJy}$ is less than $0.37 \mathrm{deg}^{-2}$ (at a confidence level of 95\%). This result is approximately an order of magnitude below the transient rate measured at $5 \mathrm{GHz}$ by Bower et al. but it is consistent with more recent upper limits from Frail et al. Our findings suggest that the radio sky at $1.4 \mathrm{GHz}$ is relatively quiet. For multi-wavelength transient searches, such as the electromagnetic counterparts to gravitational waves, this frequency may be optimal for reducing the high background of false positives.
\end{abstract}

Key words: catalogs - radio continuum: galaxies - surveys

Online-only material: color figures

\section{INTRODUCTION}

For more than four decades the largest science yield of variable and transient radio emission has come from singledish radio telescopes, which have surveyed the sky for pulsed and periodic emission from pulsars and related phenomena on typical timescales of seconds to milliseconds. In contrast, interferometric imaging surveys, which are best suited to probe timescales ranging from seconds, hours, and days, have only just begun. There are a limited number of surveys of these so-called long duration transients and variables at frequencies of 1-10 GHz, each with a different sensitivity, cadence, and field of view (FoV; Ofek et al. 2011; Bell et al. 2011). Fully exploring this phase space is one of the main science drivers for a new generation of synoptic radio imaging facilities, such as Australian Square Kilometer Array Pathfinder (ASKAP; Johnston et al. 2008) and Apertif/WSRT (Oosterloo et al. 2010), that are being built in the coming years (see also Lazio et al. 2009).

Our knowledge of the variable $\mathrm{GHz}$ sky is especially lacking at sub-milliJansky (sub-mJy) flux density levels. For persistent sources, there is a well-known flattening of the Euclideannormalized radio-source counts below about $1 \mathrm{mJy}$, corresponding to a change in the radio-source populations. This flattening is likely due to the emerging importance of star-forming galaxies and low-luminosity active galactic nuclei (AGNs) at redshifts of the order of unity (Condon 2007; Seymour et al. 2008; Smolčić et al. 2008; Padovani 2011; Condon et al. 2012). Thus, while the variability studies above mJy levels are dominated by radioloud AGNs with compact, flat-spectrum components (Sadler et al. 2006; Lovell et al. 2008), variability at these deeper flux density levels may probe new source populations.

There are indications that the mJy transient sky is exciting. Over the last decade astronomers have detected transient deci- metric emission from a variety of sources: transient, bursting and pulsed radio emission from magnetars (Cameron et al. 2005; Gaensler et al. 2005; Camilo et al. 2006), short-lived radio afterglows of short-duration gamma-ray bursts (Berger et al. 2005), emission from a (transient) jet in a dwarf nova (Körding et al. 2008), a new population of sub-relativistic supernovae (Soderberg et al. 2010), a mysterious population of bursting radio sources (Hyman et al. 2005, 2009), and relativistic outflows from tidal disruption events (Zauderer et al. 2011; Cenko et al. 2012). However, with few exceptions, most of what we know about the transient radio sky has come via radio follow-up of objects identified by synoptic telescopes at optical, $\mathrm{X}$-ray, or gamma-ray wavelengths. Clearly, this titillating trove of (serendipitous) discoveries calls for systematic exploration of the decimetric sky on timescales of minutes and longer.

A ready source of archival data for searching for sub-mJy transients and variables comes from deep continuum imaging surveys of the $\mathrm{GHz}$ radio sky undertaken to study the extragalactic radio-source populations. Many such surveys have been carried out, reaching noise levels of 4-10 $\mu \mathrm{Jy}$ and with arcsecond resolution (e.g., Schinnerer et al. 2007; de Zotti et al. 2010). In order to reach these deep flux density limits, it is standard to observe these fields for many epochs with a cadence that samples timescales of days, weeks, and months. An added benefit is that these deep surveys are accompanied by rich multi-wavelength continuum data sets and optical/infrared spectroscopic measurements. Thus, the counterpart of any unusual variable or transient source can be readily identified and its redshift determined.

In this paper, we present a search for transients and variables at sub-mJy flux density levels using data taken as part of a deep radio continuum survey toward a region known as the Extended Chandra Deep Field-South (E-CDFS; Miller et al. 2008). In Section 2, we describe the original survey and our re-reduction 


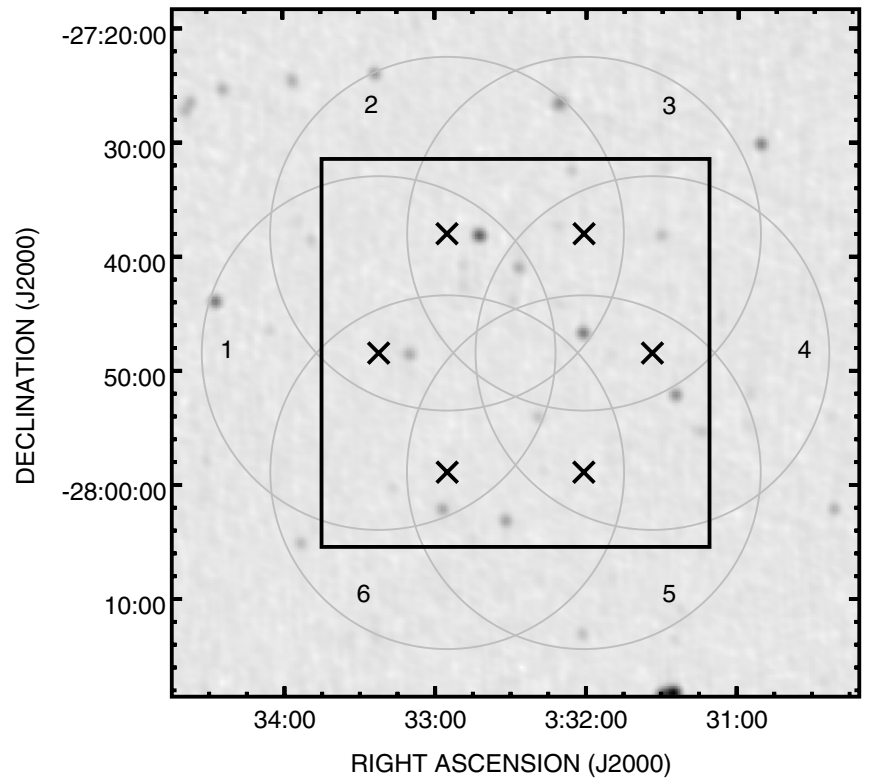

Figure 1. Hexagonal grid of six pointings (crosses), each pointing separated $12^{\prime}$ from its nearest neighbor. The $34^{\prime} \times 34^{\prime}$ (black square) region represents the extent of the final image from the Miller et al. (2008) data release. 15' circles (gray) corresponding to the $50 \%$ beam attenuation in different pointings are also shown. The $60^{\prime} \times 60^{\prime}$ background image is from NVSS.

Table 1

List of Survey Pointings

\begin{tabular}{lccr}
\hline \hline Pointing ID & R.A. (J2000.0) & Decl. (J2000.0) & $N_{\text {ep }}$ \\
\hline ECDFS1 & 033322.25 & -274830.0 & 7 \\
ECDFS2 & 033255.12 & -273803.0 & 9 \\
ECDFS3 & 033200.88 & -273803.0 & 8 \\
ECDFS4 & 033133.75 & -274830.0 & 8 \\
ECDFS5 & 033200.88 & -275857.0 & 9 \\
ECDFS6 & 033255.12 & -275857.0 & 8 \\
\hline
\end{tabular}

Note. $N_{\text {ep }}$ is the number of epochs per pointing.

of the data. In Section 3, we describe how the variability light curves for 599 point sources were extracted. The transient search is described in Section 4. The interpretation and implication of these results for radio-source variability and transients is discussed in Section 5.

\section{OBSERVATIONS AND DATA REDUCTION}

The E-CDFS is an intensely studied region with a plethora of available multi-wavelength data (viz. X-ray, ultraviolet, optical, infrared, and radio; see Miller et al. 2008 and references therein). Here, we use data from the radio survey undertaken by Miller et al. (2008). ${ }^{5}$ The observations were made at a frequency of $1.4 \mathrm{GHz}$, using the National Radio Astronomy Observatory (NRAO) Very Large Array (VLA) in its A configuration (Project code AM 889). A hexagonal grid of six pointings was made, with each pointing separated $12^{\prime}$ from its nearest neighbor (see Table 1 and Figure 1). All the observations were carried out in 2007 between June 15 and September 23. Only a single pointing was observed for each epoch. Each epoch was a $5 \mathrm{hr}$ track centered on 03:30 LST. ${ }^{6}$ There were a total of 49 epochs,

\footnotetext{
5 In the past, radio observations of this field have also been carried out by Kellerman et al. (2008), Afonso et al. (2006), and Norris et al. (2006).

6 This is of great benefit to variability studies, which are otherwise plagued by changes in the observing setup.
}

Table 2

Observing Epochs

\begin{tabular}{|c|c|c|c|}
\hline Epoch & $\begin{array}{l}\text { Date } \\
\text { UT }\end{array}$ & Pointing & $\begin{array}{c}\sigma_{\mathrm{rms}} \\
\left(\mu \mathrm{Jy} \mathrm{beam}^{-1}\right)\end{array}$ \\
\hline 1 & 2007 Jun 15 & ECDFS2 & 26.3 \\
\hline 2 & 2007 Jun 24 & ECDFS3 & 28.0 \\
\hline 3 & 2007 Jun 25 & ECDFS4 & 28.6 \\
\hline 4 & 2007 Jul 1 & ECDFS6 & 25.9 \\
\hline 5 & 2007 Jul 6 & ECDFS5 & 29.2 \\
\hline 6 & 2007 Jul 12 & ECDFS1 & 26.6 \\
\hline 7 & 2007 Jul 13 & ECDFS2 & 26.6 \\
\hline 8 & 2007 Jul 14 & ECDFS3 & 25.5 \\
\hline 9 & 2007 Jul 15 & ECDFS4 & 26.4 \\
\hline 10 & $2007 \mathrm{Jul} 16$ & ECDFS5 & 26.8 \\
\hline 11 & 2007 Jul 17 & ECDFS6 & 26.1 \\
\hline 12 & 2007 Jul 19 & ECDFS1 & 26.0 \\
\hline 13 & 2007 Jul 20 & ECDFS2 & 34.2 \\
\hline 14 & $2007 \mathrm{Jul} 21$ & ECDFS3 & 26.0 \\
\hline 15 & $2007 \mathrm{Jul} 22$ & ECDFS4 & 25.3 \\
\hline 16 & 2007 Jul 23 & ECDFS5 & 27.8 \\
\hline 17 & $2007 \mathrm{Jul} 24$ & ECDFS6 & 27.5 \\
\hline 18 & $2007 \mathrm{Jul} 26$ & ECDFS5 & 31.8 \\
\hline 19 & 2007 Jul 27 & ECDFS2 & 27.2 \\
\hline 20 & 2007 Jul 28 & ECDFS3 & 30.5 \\
\hline 21 & $2007 \mathrm{Jul} 29$ & ECDFS4 & 27.1 \\
\hline 22 & 2007 Jul 30 & ECDFS1 & 28.0 \\
\hline 23 & 2007 Aug 2 & ECDFS6 & 27.0 \\
\hline 24 & 2007 Aug 3 & ECDFS1 & 27.4 \\
\hline 25 & 2007 Aug 4 & ECDFS2 & 26.7 \\
\hline 26 & 2007 Aug 5 & ECDFS3 & 25.9 \\
\hline 27 & 2007 Aug 6 & ECDFS4 & 29.1 \\
\hline 28 & 2007 Aug 9 & ECDFS5 & 31.2 \\
\hline 29 & 2007 Aug 10 & ECDFS6 & 30.0 \\
\hline 30 & 2007 Aug 11 & ECDFS1 & 31.9 \\
\hline 31 & 2007 Aug 13 & ECDFS2 & 31.3 \\
\hline 32 & 2007 Aug 14 & ECDFS3 & 31.5 \\
\hline 33 & 2007 Aug 16 & ECDFS4 & 30.8 \\
\hline 34 & 2007 Aug 17 & ECDFS5 & 45.7 \\
\hline 35 & 2007 Aug 18 & ECDFS6 & 28.6 \\
\hline 36 & 2007 Aug 21 & ECDFS1 & 29.1 \\
\hline 37 & 2007 Aug 23 & ECDFS2 & 30.9 \\
\hline 38 & 2007 Aug 25 & ECDFS3 & 30.8 \\
\hline 39 & 2007 Aug 26 & ECDFS4 & 29.8 \\
\hline 40 & 2007 Aug 28 & ECDFS5 & 33.9 \\
\hline 41 & 2007 Aug 31 & ECDFS6 & 29.3 \\
\hline 42 & 2007 Sep 6 & ECDFS2 & 30.9 \\
\hline 43 & 2007 Sep 7 & ECDFS3 & 29.3 \\
\hline 44 & 2007 Sep 8 & ECDFS4 & 29.3 \\
\hline 45 & 2007 Sep 9 & ECDFS5 & 28.9 \\
\hline 46 & 2007 Sep 10 & ECDFS6 & 30.2 \\
\hline 47 & 2007 Sep 11 & ECDFS1 & 30.4 \\
\hline 48 & 2007 Sep 12 & ECDFS5 & 37.9 \\
\hline 49 & 2007 Sep 23 & ECDFS2 & 31.6 \\
\hline
\end{tabular}

Notes. List of the 49 epochs. Each epoch consisted of a $5 \mathrm{hr}$ track centered on 03:30 LST.

with a combined allocation of $245 \mathrm{hr}$ (see Table 2). The total number of epochs for each pointing $N_{\mathrm{ep}}$ is given in Table 1. For more details about the specifics of the observational setup, see Miller et al. (2008).

The original purpose of these data was to average together all pointings and epochs in order to create a deep $\left(\sigma_{\mathrm{rms}}=5-8 \mu \mathrm{Jy}\right)$ continuum image of the E-CDFS. In order to explore variability and to search for transients, we needed to work with the single epoch, single pointing images instead. Despite this, many of the data reduction steps that we followed were similar to Miller 


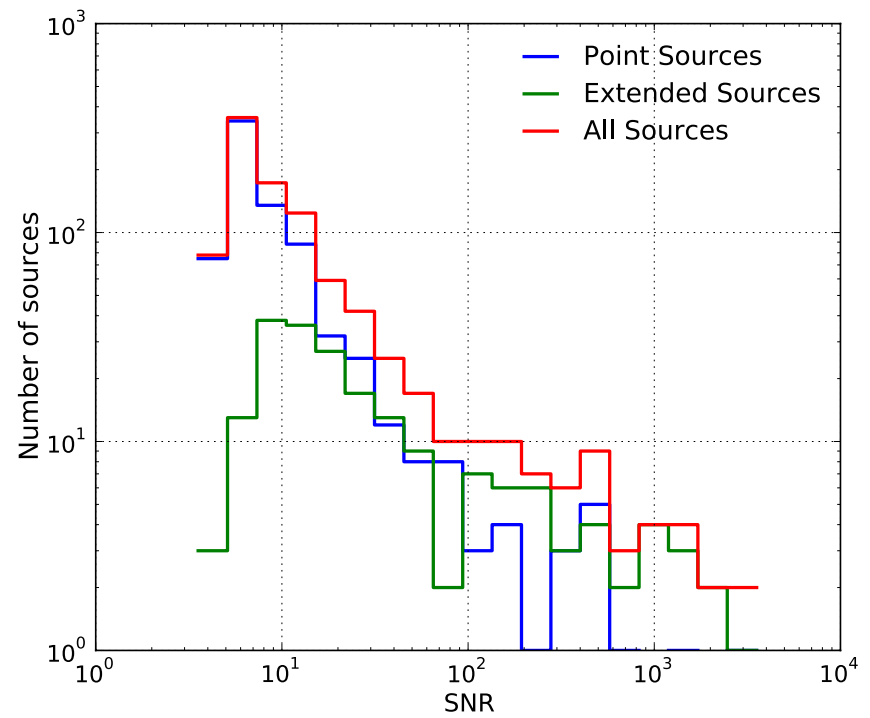

Figure 2. Histogram of the $\mathrm{S} / \mathrm{N}$ of the sources in the DR2 catalog. (A color version of this figure is available in the online journal.)

et al. (2008). We summarize the process here, pointing out slight differences. All calibration and imaging was carried out in the Astronomical Image Processing System (AIPS) package. ${ }^{7}$

The calibrated $u v$ data for each epoch were imaged and deconvolved separately. In order to image the entire FoV at full resolution, we created 37 different facets in a flys-eye pattern. Each of the 37 facets was an image of $1024^{2}$ pixels with a 0.5 pixel size offset from one another. There were another 23-25 outlier fields of $128^{2}$ pixels each made of cataloged bright radio sources outside the primary beam of each pointing but within a $2^{\circ}$ radius.

The AIPS task IMAGR was used to deconvolve each image to the rms noise level. In order to have a consistent set of images, we applied a Gaussian taper to the (30\% level) of $100 \mathrm{k} \lambda$ and 70 $\mathrm{k} \lambda$ in the $u$ and $v$ directions to the visibility data, and we restored the final images to a synthesized beam of $2^{\prime \prime} .8 \times 1^{\prime \prime} .6$ (position angle $\simeq 0^{\circ}$ ). After deconvolution, the 37 facets for each epoch were combined using the AIPS task FLATN to form a single $5120^{2}$ pixel image $42^{\prime} .7$ across. A correction for the attenuation from the primary beam was not applied at this stage in order that the images used in analysis had uniform noise statistics across the entire image. The rms noise $\sigma_{\mathrm{rms}}$ for each epoch is given in Table 2.

\section{VARIABILITY ANALYSIS}

The source catalog we used to investigate variability was taken from the second data release (DR2; Miller et al. 2013) of Miller et al. (2008). This catalog was generated by combining all the data from Tables 1 and 2 to make a single deep $34^{\prime} \times 34^{\prime}$ image with a typical sensitivity of $7.4 \mu \mathrm{Jy}$. Miller et al. (2013) identified sources using the AIPS task SAD down to $4 \sigma$, and then inspected the residual map to identify missed sources as well as accepted sources which were poorly fit by SAD. These missing sources were then added to the preliminary source list. Further flagging and follow-up was done in order to produce a modified source list in which all sources with peak flux density greater than five times the local rms noise (i.e., $5 \sigma$ ) were fit using the AIPS task JMFIT. Also, the effect of bandwidth smearing on

\footnotetext{
7 http://www.aips.nrao.edu/
}

sources within the six individual pointings was assessed using JMFIT, and the resolution information was thus preserved in the output catalog. Lastly, the sources in the DR2 catalog were compared with the catalog of Kellerman et al. (2008).

The DR2 catalog contains almost twice as many sources ( 883 versus 464) compared to the first data release (see Miller et al. $2008,2013)$, owing to a more careful data reduction. Of the 883 sources in the DR2 catalog, we created a point-source-only catalog of 736 objects used for exploring variability. With this careful approach outlined above, we expect the Miller et al. DR2 catalog to contain all real sources above $5 \sigma$ (however, see Section 4.1).

We thus justify our use of the DR2 catalog for investigating the variability of the sub-mJy population. In Section 4, we use both the DR2 image and its source catalog as a test bed for different source-finding algorithms. The signal-to-noise ratio $(\mathrm{S} / \mathrm{N}$; denoted as SNR in the figures) of sources in the DR2 catalog is shown in Figure 2.

It is important to quantify the uncertainty in the peak flux density when analyzing the variability of sources in different epochs. Following Thyagarajan et al. (2011), we attribute this uncertainty primarily to a combination of four ${ }^{8}$ causes: (i) local image rms noise, (ii) uncertainty in the primary beam, (iii) flux density calibration amplitude, and (iv) pointing errors. Let us denote the measured peak flux density of a source in epoch $i$ by $f_{i}$, such that the primary beam correction is $b=b(\theta)$, where $\theta$ is the angular distance of the source from the phase center. We wish to calculate the uncertainty in the quantity $\left(f_{i} / b\right)$. The image local noise $\left(\Delta f_{i}\right)$ scales as $\Delta f_{i} / b$. If we denote the fractional uncertainty in the beam as $\epsilon_{b}$ and that in the flux density calibration amplitude by $\epsilon_{c}$, then the corresponding errors scales as $\left(f_{i} / b\right) \epsilon$. We adopt a value of $4 \%$ for $\epsilon_{c}$, intermediate between the conservative estimate of Thyagarajan et al., 5\%, and the one quoted by Ofek et al. (2011), 3\%. The typical pointing error $(\Delta \theta)$ of a VLA antenna is between $10^{\prime \prime}$ and $20^{\prime \prime}$. The resultant uncertainty scales as $\left(f_{i} / b^{2}\right)(-d b / d \theta) \Delta \theta$. All four of these error terms, added in quadrature, would give the total uncertainty, $\sigma_{i}$, in the peak flux density corrected for the primary beam attenuation. However, as shown below, the pointing-related error term is much smaller than the rest, and hence can be neglected. Thus,

$$
\sigma_{i}=\frac{1}{b} \sqrt{\Delta f_{i}^{2}+f_{i}^{2}\left(\epsilon_{b}^{2}+\epsilon_{c}^{2}\right)} .
$$

Polynomial coefficients (and the associated error) that express the average angular dependence of $b$ can be found in the AIPS task PBCOR, while measurements of the VLA beam power response to beyond the first null are given in Cotton \& Perley (2010). Using the beam response profile from Cotton \& Perley, we can estimate the error terms (i)-(iv) above for a typical source in the DR2 catalog, having measured a flux density of $300 \pm 30 \mu \mathrm{Jy}$. If the source lies at the half-power radius $\left(\theta=15^{\prime}\right)$, then these correction factors are about $10 \%, 4 \%, 4 \%$, and $1 \%$ of the primary-beam-corrected flux density $(570 \mu \mathrm{Jy})$, respectively. The pointing-related uncertainty is thus negligible.

In light of Ofek et al. (2011), we use two measures of variability (see also Scheers 2011), the modulation index defined

\footnotetext{
8 Far out in the primary beam, orthogonally polarized beams can be far offse on the sky, making amplitude calibration difficult. For short observations, the effects of this "beam squint" can be much larger than all other uncertainties combined. However, these offsets tend to average out when observations are made over sufficiently long times. Since the VLA's beam squint is oriented almost exactly east-west (Cotton \& Perley 2010), our observational setup is optimal for averaging out the effects of beam squint.
} 


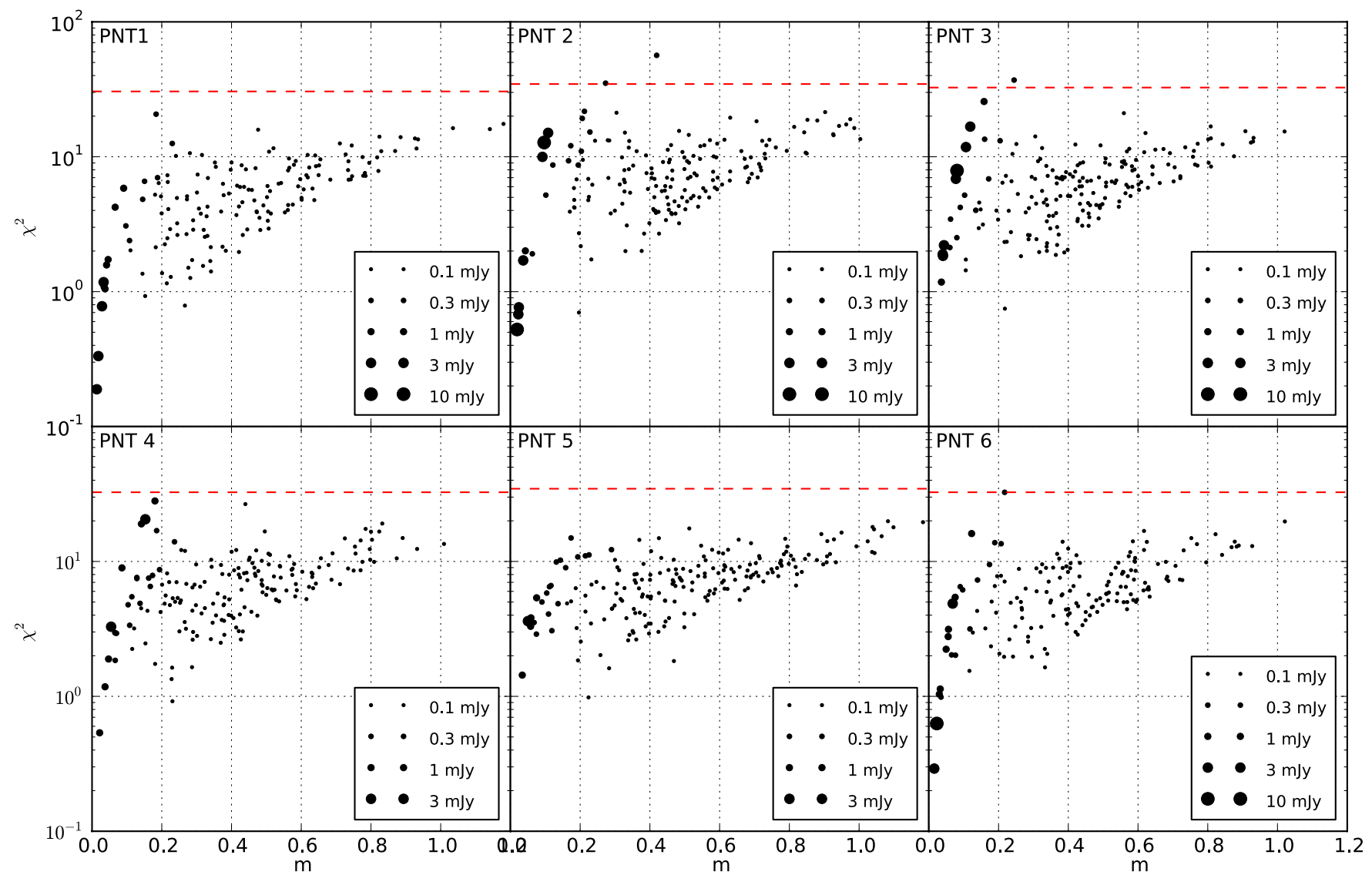

Figure 3. Variability plot for the point sources in the Miller et al. DR2 catalog, shown separately for all pointings. The peak flux density is denoted by the symbol size. The red dashed line represents the $4 \sigma$ level for the appropriate number of degrees of freedom (one less the number of epochs in each pointing) for each pointing. The number of epochs in each pointing lies between 7 and 9.

(A color version of this figure is available in the online journal.)

as the standard deviation divided by the mean,

$$
m=\frac{1}{\bar{f}} \sqrt{\frac{1}{\mathrm{~N}-1} \sum_{i=1}^{N}\left(f_{i}-\bar{f}\right)^{2}},
$$

and the $\chi^{2}$,

$$
\chi^{2}=\sum_{i=1}^{N} \frac{\left(f_{i}-\bar{f}\right)^{2}}{\sigma_{i}^{2}},
$$

where $N$ is the number of epochs, and $\bar{f}$ is the mean flux density of the source over all the epochs considered for the variability analysis. In these two equations, the primary beam correction is implicit in $f_{i}$ and $\bar{f} . \chi^{2}$ gives a measure of the deviation from stochastic epoch-to-epoch fluctuations in the peak flux density, and we define "significant variability" beyond a level" of $4 \sigma$. The modulation index indicates the strength of variability, i.e., the fractional variation of the peak flux density.

\subsection{Single-pointing Variability}

A variability analysis was carried out on each pointing in Table 1 separately. Peak flux densities were measured for all point sources in the DR2 catalog brighter than $40 \mu \mathrm{Jy}$ and within

9 For Gaussian noise, $4 \sigma$ corresponds to a probability of about $1 / 16,000$, while the number of measurements in our variability analysis (several to tens of epochs multiplied by a few hundred sources) ranges from about 1500 to 15,000 . a $15^{\prime}$ radius of the pointing centers (i.e., the $50 \%$ response radius of the primary beam of the VLA antennas; see also Figure 1). This approach has the merit of being simple and robust. Since the angular distance of a source from its pointing center is constant, the accuracy of the correction for the primary beam attenuation $b(\theta)$ is unimportant. The modulation index and $\chi^{2}$ measures of variability are insensitive to a constant $b(\theta)$.

The limitation of this approach is that the resulting light curves are constructed for only 7-9 epochs. Many of our investigated point sources are found in multiple pointings and therefore light curves can be constructed with many more epochs, resulting in higher cadence over the full 100 days of observing. A full variability analysis of this kind is carried out in Section 3.2.

In Figure 3, we show variability plots (i.e., $\chi^{2}$ versus $m$ ) for the DR2 sources in each of the six pointings. There are approximately 175 sources per pointing. Some of the bright ( $>3 \mathrm{mJy}$ ) sources show significant variability but with only low modulation indices $(\sim 10 \%)$. We define strong variables as sources having $m>0.5$ (i.e., higher than $50 \%$ fractional variability). Only two genuine variables were found in this single-pointing analysis; no strong variables were found (Table 3, upper panel).

\subsection{Full Variability Analysis}

In order to undertake variability analysis using data from all the epochs, the single-epoch peak flux densities were required to be corrected for (i) bandwidth smearing, and (ii) the primary beam response, as any given source will lie at a different angular 
Table 3

Variables Among Miller et al. DR2 Sources

\begin{tabular}{|c|c|c|c|c|c|c|c|c|c|c|c|c|}
\hline ID & $\begin{array}{c}\alpha_{J 2000} \\
(\mathrm{~h}, \mathrm{~m}, \mathrm{~s})\end{array}$ & $\begin{array}{l}\delta_{J 2000} \\
\left({ }^{\circ},{ }^{\prime},{ }^{\prime \prime}\right)\end{array}$ & $\begin{array}{c}\bar{f} \\
(\mu \mathrm{Jy})\end{array}$ & $m$ & $\begin{array}{l}V_{c} \\
(\%)\end{array}$ & $N_{\mathrm{ep}}$ & $z$ & $\begin{array}{c}\log \bar{L}_{R} \\
(\mathrm{cgs})\end{array}$ & $\alpha_{\mathrm{R}}$ & $\alpha_{\mathrm{IR}}$ & $\begin{array}{c}M_{R} \\
(\mathrm{mag})\end{array}$ & $\begin{array}{l}\text { Energy } \\
\text { Source }\end{array}$ \\
\hline \multicolumn{13}{|c|}{ Single Pointing Analysis } \\
\hline 337 & 033218.03 & -274718.8 & $558 \pm 14$ & 0.26 & 76 & 8 & & & & & & \\
\hline 621 & 033315.00 & -275151.3 & $663 \pm 22$ & 0.23 & 93 & 9 & & & & & & \\
\hline \multicolumn{13}{|c|}{ Full Variability Analysis } \\
\hline 67 & 033127.07 & -274409.9 & $136 \pm 9$ & 0.50 & 181 & 16 & 1.005 & 30.6 & +0.23 & $>-3.8$ & -21.04 & $\mathrm{SF}+\mathrm{AGN}$ \\
\hline 190 & 033152.13 & -273926.6 & $891 \pm 12$ & 0.15 & 48 & 16 & 2.296 & 32.1 & -0.35 & $>-1.3$ & -22.07 & AGN \\
\hline 239 & 033200.85 & -273557.1 & $1822 \pm 27$ & 0.12 & 38 & 8 & 0.266 & 30.5 & -0.30 & $>-2.3$ & -17.26 & $\mathrm{SF}+\mathrm{AGN}$ \\
\hline 297 & 033211.66 & -273726.3 & $3600 \pm 36$ & 0.11 & 41 & 17 & 0.605 & 31.5 & +0.89 & -0.9 & -23.98 & AGN \\
\hline 337 & 033218.03 & -274718.8 & $494 \pm 11$ & 0.26 & 95 & 16 & 0.734 & 30.8 & -0.08 & $>-3.4$ & -22.69 & $\mathrm{SF}+\mathrm{AGN}$ \\
\hline 621 & 033315.00 & -275151.3 & $497 \pm 10$ & 0.20 & 70 & 15 & 1.107 & 31.2 & +0.24 & $>-3.4$ & -20.71 & $\mathrm{SF}+\mathrm{AGN}$ \\
\hline 628 & 033316.74 & -275630.4 & $1341 \pm 16$ & 0.11 & 38 & 15 & 0.685 & 31.2 & -0.40 & -2.2 & -21.21 & $\mathrm{SF}+\mathrm{AGN}$ \\
\hline
\end{tabular}

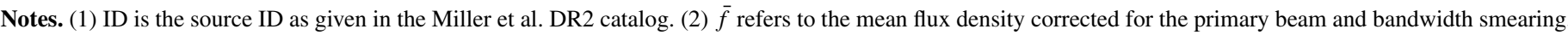

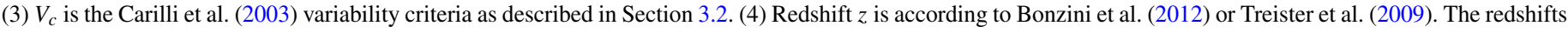

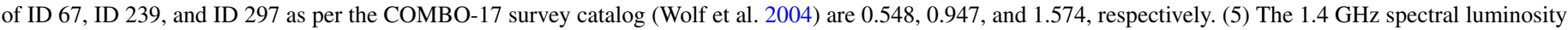

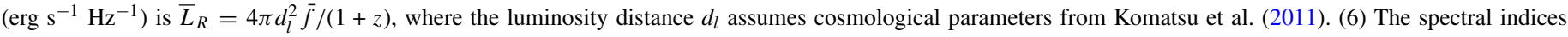

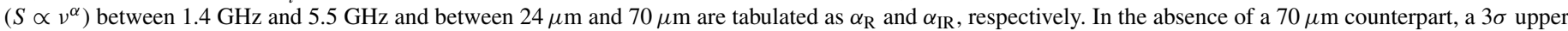

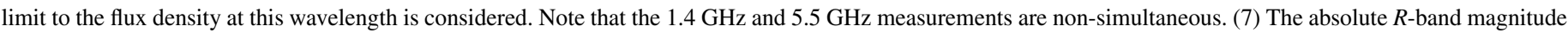

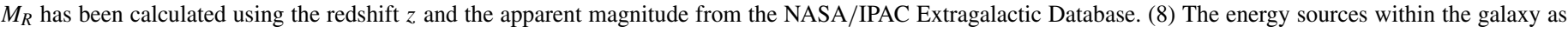
indicated by the radio and mid-to-far-infrared properties are listed in the last column. SF: star formation; AGN: active galactic nucleus (see Section 3.3).

distance from the phase center in different pointings. We applied bandwidth-smearing correction from the approximation given in Bridle \& Schwab (1989, Equation (13-19) therein).

There were two choices for the primary beam profileone derived as the empirical beam profile for VLA-FIRST (Thyagarajan et al. 2011), and the other found in the AIPS task PBCOR. To test which of these profiles best represented our data, we adopted the following approach. We first normalized the peak flux densities of $>300 \mu \mathrm{Jy}$ sources from all epochs using their peak flux density from the DR2 catalog and plotted them as a function of distance from the pointing center. The resultant beam profile matched with the VLA-FIRST profile better than the one from PBCOR (to within 1\%, but only for $\theta<12^{\prime}$; scatter of $6.5 \%$ ). Hence, we used the former beam profile for our primary beam correction, $b(\theta)$; the associated error $\left(\epsilon_{b}\right)$ was also taken from Thyagarajan et al. (2011). Thus, for a reliable all-epoch variability analysis, we restricted our search to the point sources in the DR2 catalog which were located within $12^{\prime}$ from the pointing centers of their respective epochs. This also appears to be the radius beyond which our bandwidth-smearing approximation starts to break down. Thus, for example, a source located at $\alpha=03^{\mathrm{h}} 33^{\mathrm{m}} 00^{\mathrm{s}}$ and $\delta=-28^{\circ} 00^{\prime} 00^{\prime \prime}$ would be present in pointings 6,5 , and 1 , but not in 2,3 , and 4 because the separation between the source and the centers of pointings 2, 3, and 4 is larger than 12 arcmin. Further, as we did with the single-pointing variability (Section 3.1), we restricted our analysis to sources whose mean flux density was brighter than $40 \mu \mathrm{Jy}$. This full variability analysis was carried out on 599 point sources.

Depending on the number of pointings in which a source is present, this analysis allowed us to exploit the higher cadence over the entire duration of the observing program. The resulting light curves are now more densely sampled with 15-26 epochs, rather than the 7-9 epochs for the single-pointing variability case. In Figure 4, we show variability plots for the DR2 sources, taking into account all the epochs.

Seven significant variables were found via this procedure, but no strong variables $(m>0.5)$. Both of the variables identified

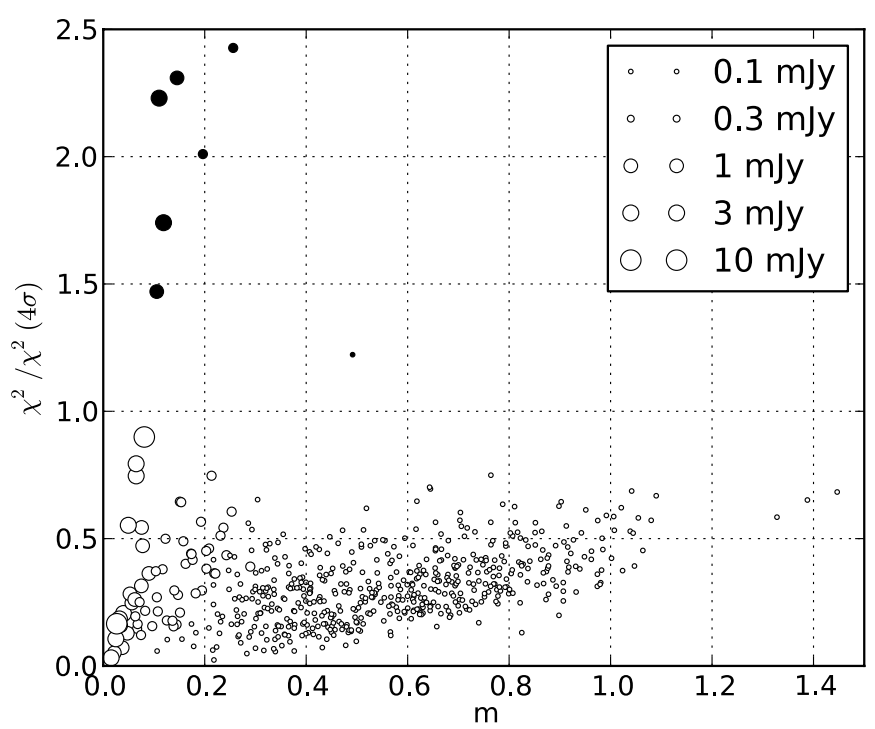

Figure 4. $\chi^{2}$ normalized by its value at the $4 \sigma$ level, plotted against the modulation index, $m$, for sources in the Miller et al. DR2 catalog using peak fluxes from all pointings, and corrected with empirically derived beam attenuation profile. The $4 \sigma$ level is different for different sources, depending on the number of epochs in which they are present. The mean peak flux density is denoted by the symbol size. Filled circles indicate significant variables (lying above a normalized $\chi^{2}$ of unity).

in the single-pointing analysis in Section 3.1 are also seen here. The results of the variability study are given in Table 3 and the light curves for the significant variables are shown in Figure 5. We can compare our variability criteria with the Carilli et al. (2003) measure for variability, i.e., $V_{c}=\left(S_{1}-S_{2}\right) / S$, where $S_{1}$ and $S_{2}$ are the maximum and minimum flux densities observed, respectively, and $S$ is their mean. This measure of variability for the seven variable sources found in this work are listed in Table 3.

In order to undertake multi-wavelength identifications, we had to align the reference frames of all the data sets. The radio and optical source positions were brought to the same 

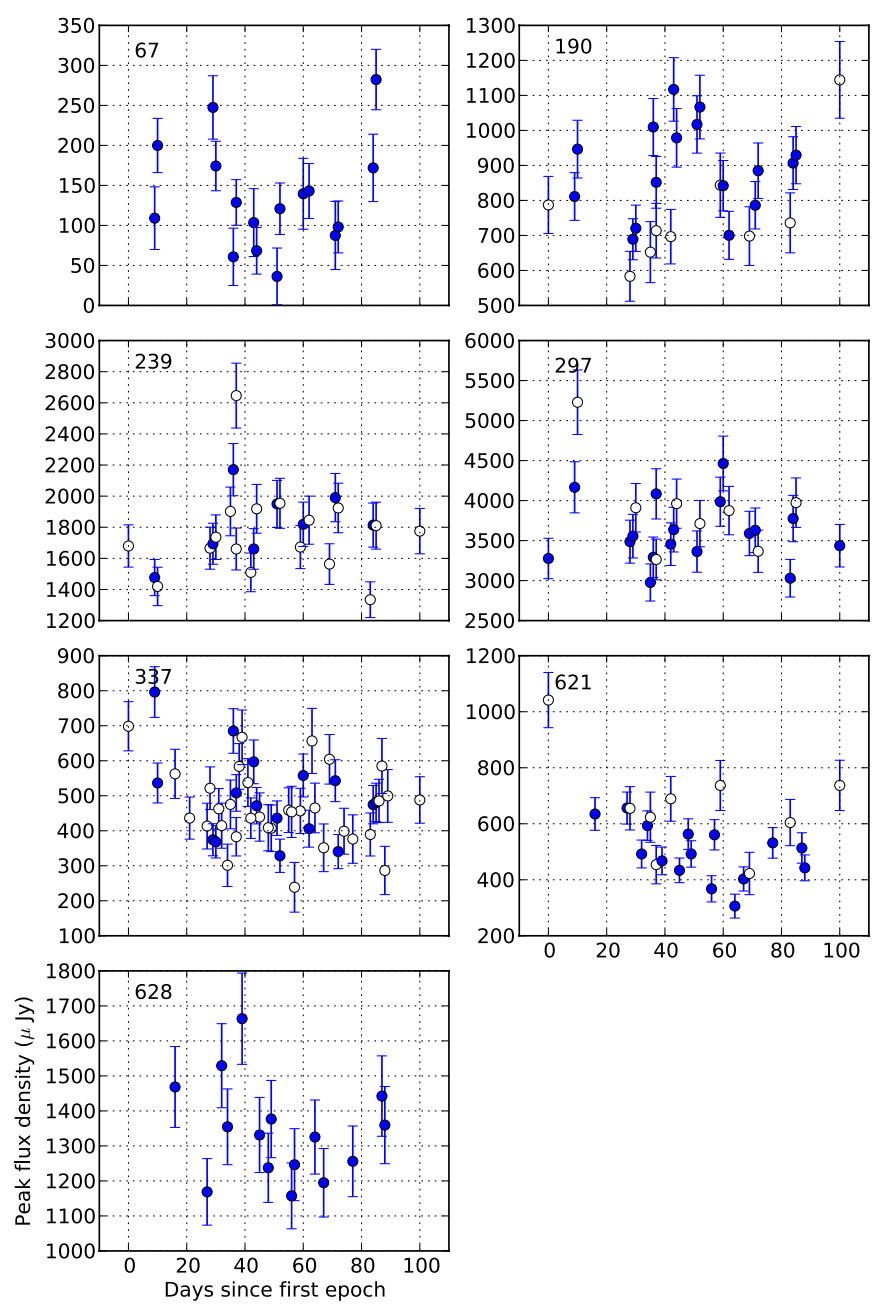

Figure 5. Light curves of the variable sources from Miller et al. DR2 catalog listed in Table 3. The filled symbols represent the flux densities considered for variability analysis (i.e., where $\theta<12^{\prime}$ ). Flux densities in epochs where a source lies within the $50 \%$ power circle of the beam (i.e., where $\theta \lesssim 15^{\prime}$ ) are plotted for reference as open symbols. The error bars take into account the background rms, primary beam correction, and bandwidth smearing (no taper). (A color version of this figure is available in the online journal.)

reference frame by calculating the radio-source position offsets with respect to Hubble Space Telescope (HST) source positions from GOODS-S (Giavalisco et al. 2004) and the GEMS (Rix et al. 2004). Optical counterparts (from these two HST catalogs) were searched toward radio sources within $1^{\prime \prime}$. Only point-like sources having a single counterpart were chosen. A histogram of the offsets of these counterparts in right ascension $(\Delta \alpha=$ $\left.\alpha_{\text {radio }}-\alpha_{\text {optical }}\right)$ and declination $\left(\Delta \delta=\delta_{\text {radio }}-\delta_{\text {optical }}\right)$ was then computed to find the most likely offset (peak of the histogram). The associated error was taken to be the standard deviation about this most likely offset added in quadrature with the cataloged mean positional error of the radio source. In addition to the Miller et al. (2013) DR2 catalog, we repeated this procedure for other radio-source catalogs in the E-CDFS region, viz., Kellerman et al. (2008), Norris et al. (2006), and Afonso et al. (2006) using $\geqslant 5 \sigma$ sources. The computed radio versus optical positional offsets along with the mean positional errors listed in the respective radio catalogs are shown in Table 4.

\subsection{Notes on Variables Found}

An important question that we can address is whether the variability at sub-mJy levels is dominated by normal star-
Table 4

Radio Positional Offsets with Respect to Optical HST Catalogs

\begin{tabular}{cccccc}
\hline \hline & $\operatorname{arcsec}$ & $(1)$ & $(2)$ & $(3)$ & $(4)$ \\
\hline & $\sigma_{\alpha}$ & $\sim 0.1$ & 0.37 & 0.31 & $\mathrm{~b}$ \\
& $\sigma_{\delta}$ & $\sim 0.1$ & 0.57 & 0.58 & $\mathrm{~b}$ \\
\hline \multirow{2}{*}{ GEMS } & $\Delta \alpha$ & $0.18 \pm 0.31$ & $0.15 \pm 0.45$ & $-0.09 \pm 0.41$ & $-0.17 \pm 0.40^{\mathrm{a}}$ \\
& $\Delta \delta$ & $-0.32 \pm 0.32$ & $-0.34 \pm 0.53$ & $-0.20 \pm 0.44$ & $0.11 \pm 0.30^{\mathrm{a}}$ \\
\hline \multirow{2}{*}{ GOODS } & $\Delta \alpha$ & $-0.20 \pm 0.23$ & $0.15 \pm 0.42^{\mathrm{a}}$ & $0.03 \pm 0.44^{\mathrm{a}}$ & $-0.10 \pm 0.39^{\mathrm{a}}$ \\
& $\Delta \delta$ & $0.22 \pm 0.28$ & $-0.18 \pm 0.37^{\mathrm{a}}$ & $0.03 \pm 0.44^{\mathrm{a}}$ & $-0.18 \pm 0.33^{\mathrm{a}}$
\end{tabular}

Notes. Columns: (1) Miller et al. (2013); (2) Kellerman et al. (2008); (3) Norris et al. (2006); (4) Afonso et al. (2006). All offsets are in arcseconds.

${ }^{a}$ Few $(\leqslant 15)$ sources available to calculate the offsets.

b Positional uncertainty not mentioned in catalog; assumed to be 0.1 .

forming galaxies or by AGNs. For AGN-dominated samples above $1 \mathrm{mJy}$, variability at frequencies of a few $\mathrm{GHz}$ or below is thought to be dominated by propagation effects (i.e., refractive interstellar scintillation) and not by intrinsic changes in the source (Gaensler \& Hunstead 2000; Ofek \& Frail 2011). For a disk galaxy, we expect there to be steady emission from diffuse synchrotron emission and the sum of all supernovae. Intrinsic variability can be induced by (i) a stellar explosion (supernova, low-luminosity gamma-ray burst), and (ii) nuclear radio emission (AGNs). Mapping the radio emission to the center of the galaxy would favor (ii), whereas if the radio emission is mapped to the disk then (i) is favored.

To this end, we overplotted radio-source positions of the seven variables given in Table 3 on HST image cutouts from GOODS-S and the GEMS projects (Figure 6). All of the radio sources have an optical counterpart on these HST images. The redshifts of these objects vary from 0.3 to 2.3 . Thus, the postoffset radio-source position uncertainties lie between 1.4 and $2.7 \mathrm{kpc}$. Within most error ellipses, there is a centrally compact source, suggesting that this is the source of the variable emission. Light curves of the variable sources are given in Figure 5. Detailed notes on each of these objects are given below, and key physical parameters are listed in Table 3 . All of the variable sources have luminosities in excess of $10^{30} \mathrm{erg} \mathrm{s}^{-1} \mathrm{~Hz}^{-1}$, where the luminosity functions of AGNs and star-forming galaxies intersect (Condon et al. 2002). Most of the variable sources have a roughly flat spectrum between $1.4 \mathrm{GHz}$ and $5.5 \mathrm{GHz}$ suggesting the presence of AGNs. Note that the spectral indices between $1.4 \mathrm{GHz}$ and $5.5 \mathrm{GHz}$ are based on non-simultaneous measurements having different resolutions. Additionally, midinfrared colors, far-infrared spectral indices, and mid-to-farinfrared luminosities indicate that most of the variable sources reside in star-forming galaxies. In Figure 7, we show the midinfrared color-color diagram for the variable sources using Spitzer/IRAC photometry from the SIMPLE survey catalog (Damen et al. 2011). The Donley et al. (2012) AGN selection region is overplotted. For comparison, the colors of the radio sources from the AEGIS20 sample (Willner et al. 2012) are shown along with those of three template spectral energy distributions (SEDs) - an elliptical galaxy, an Sbc galaxy, and an AGN-from Assef et al. (2010). This figure along with the far-infrared flux densities identify two variables with AGNs and the rest as star-forming galaxies.

To summarize, the high-resolution optical images together with photometric information from radio and mid-to-farinfrared suggest that variability arises from the central regions of an AGN or star-forming galaxy. 

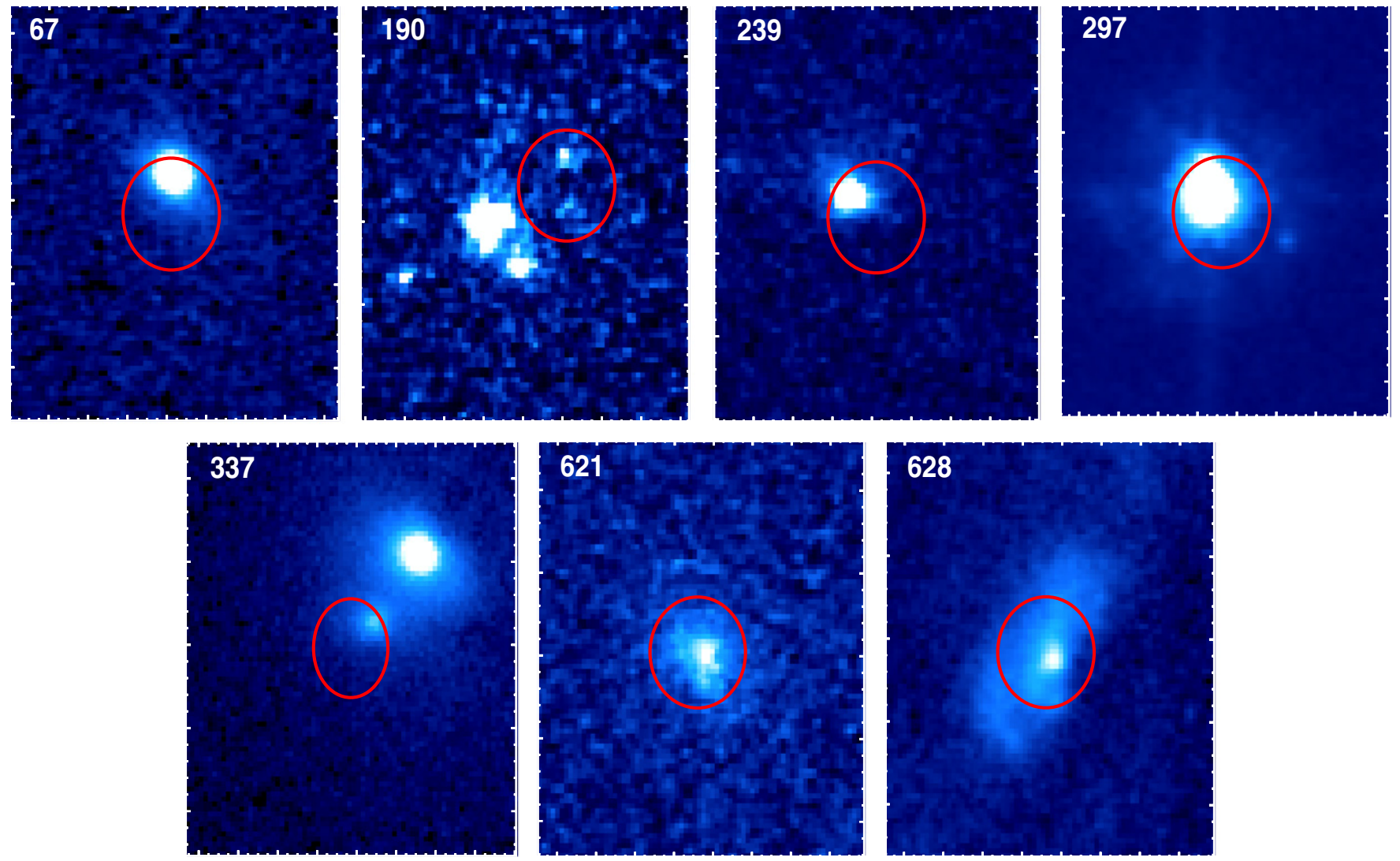

Figure 6. 2".2 2 2". 5 GEMS (all sources except ID 337) and GOODS-S (ID 337) F606W ACS-WFC image cutouts of variable sources found in this work. The red error ellipses denote the radio-source positions from Miller et al. (2013), shift-corrected to the HST source positions. All the positional uncertainties are $1 \sigma$ (see Section 3.2 and Table 4).

(A color version of this figure is available in the online journal.)

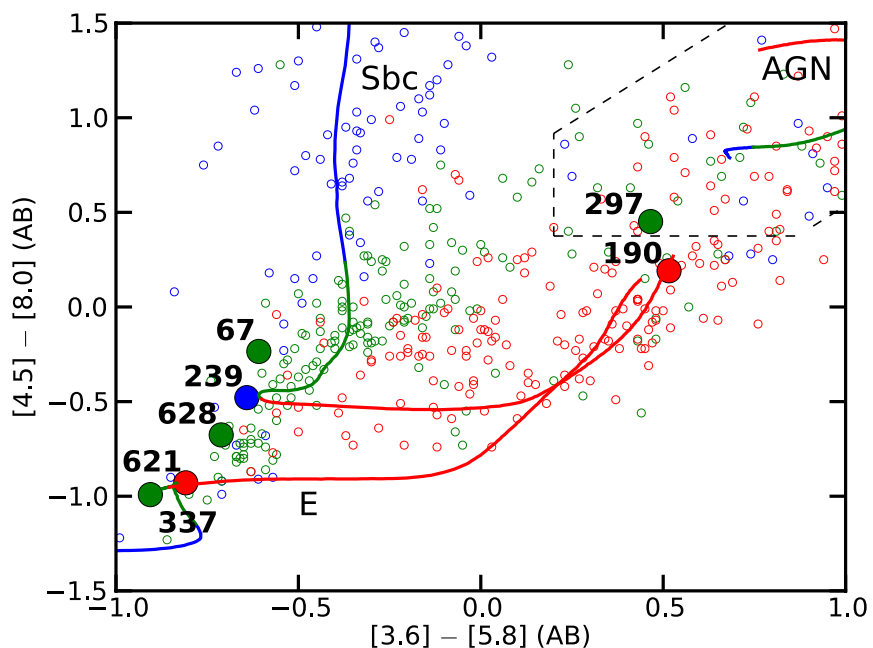

Figure 7. Spitzer/IRAC color-color diagram for the mid-infrared counterparts of the variable sources (filled circles). For comparison, the radio sources from AEGIS20 (Willner et al. 2012) are also shown (unfilled circles). The AGN selection region in the upper-right corner, bounded by the dashed lines, is from Donley et al. (2012). Curves represent the colors of three template spectral energy distributions (E: elliptical galaxy; Sbc: spiral galaxy; and AGN) from Assef et al. (2010) as redshift increases from 0 to 3 . The redshift-dependent color coding is-blue: $z \leqslant 0.5$, green: $0.5<z \leqslant 1.1$, and red: $z>1.1$.

(A color version of this figure is available in the online journal.)

ID 67. This source has a mean $1.4 \mathrm{GHz}$ flux density of $136 \pm 9 \mu \mathrm{Jy}$ and shows the strongest variation among all seven variables. Two flaring bursts are evident from the light curve
(Figure 5), one of them lasting for about 25 days, and the flux density of the other increasing by at least a factor of 2.5 in 12 days. This source also appears in the Kellerman et al. $1.4 \mathrm{GHz}$ catalog, where its flux density is $90 \pm 16 \mu \mathrm{Jy}$. The photometric redshift of $z=1.005$ (Bonzini et al. 2012) implies a mean radio luminosity of $\log L_{R}=30.6 \mathrm{erg} \mathrm{s}^{-1} \mathrm{~Hz}^{-1}$. The radio spectral index (defined as $\alpha$, where $\mathrm{S}_{v} \propto v^{\alpha}$ ) between $1.4 \mathrm{GHz}$ and $5.5 \mathrm{GHz}$ is +0.23 (Huynh et al. 2012). The morphology of the host galaxy as seen from the HST image appears to be that of a bright, compact nucleus surrounded by faint extended structure. The half-light radius of the galaxy according to the GALFIT parameters from the GEMS catalog is $620 \pm 10 \mathrm{pc}$. The $K$-corrected ${ }^{10}$ mid-to-far-infrared spectral luminosities derived from the FIDEL and GOODS (Magnelli et al. 2009, 2011), and SIMPLE Spitzer surveys are $v L_{v}(5.8 \mu \mathrm{m})=6.6 \times 10^{8} L_{\odot}$ and $v L_{v}(24 \mu \mathrm{m})<5.4 \times 10^{10} L_{\odot}$. By comparing these quantities with the Chary \& Elbaz (2001) template SEDs (see Figure 4 of that paper) and from the Spitzer/IRAC color-color diagram (Figure 7), we interpret that the host galaxy is star-forming.

Taken together, the radio luminosity, radio spectral index, optical morphology, and mid-to-far-infrared flux densities argue that ID 67 is a star-forming galaxy harboring a low-luminosity AGN.

ID 190. This source has a mean $1.4 \mathrm{GHz}$ flux density of $891 \pm 12 \mu \mathrm{Jy}$. The light curve appears to fluctuate between high- and low-flux density states on a timescale of the order

\footnotetext{
${ }^{10}$ For the $5.8 \mu \mathrm{m}$ and $24 \mu \mathrm{m}$ luminosities, the $K$-correction has been applied based on the $8 \mu \mathrm{m}$ and $70 \mu \mathrm{m}$ flux densities. In the absence of a $70 \mu \mathrm{m}$ detection, the $3 \sigma$ upper limit has been used.
} 
of 20 days. This source is also found in the Kellerman et al. and Norris et al. catalogs where the flux density is $970 \pm 30$ and $810 \pm 19 \mu \mathrm{Jy}$, respectively, with a spectral index of -0.6 between 1.4 and $4.8 \mathrm{GHz}$. The $5.5 \mathrm{GHz}$ flux density from Huynh et al. is $555 \pm 17 \mu \mathrm{Jy}$, for which we derive a spectral index of -0.35 . The photometric redshift of $z=2.296$ (Bonzini et al. 2012) implies a mean radio luminosity of $\log L_{R}=32.1$ (cgs). The HST image of this galaxy as seen in Figure 6 shows that the radio source is offset from the brightest emission in the field. On the basis of its departure from the radio-FIR correlation, Norris et al. classify this source as an AGN. The radio and far-infrared spectral indices (Table 3) and mid-infrared colors (Figure 7) are consistent with this identification. Note that between $24 \mu \mathrm{m}$ and $70 \mu \mathrm{m}$, a spectral index greater than -1.5 is representative of AGNs (e.g., Condon et al. 2002).

ID 239. This source has a mean $1.4 \mathrm{GHz}$ flux density of $1822 \pm 27 \mu \mathrm{Jy}$. This source is also found in the Kellerman et al. and Norris et al. catalogs where the flux density is $2030 \pm 43$ and $1640 \pm 20 \mu \mathrm{Jy}$, respectively. Its light curve shows a gradual increase in flux density over a period of about 50 days, followed by an equally gradual decline. The radio spectral index between $1.4 \mathrm{GHz}$ and $5.5 \mathrm{GHz}$ is -0.30 . This slope is more consistent with AGNs than star-forming galaxies which are expected to have $\alpha \simeq-0.8$. The redshift $z=0.266$ (Treister et al. 2009) implies a mean radio luminosity of log $L_{R}=30.5$ (cgs). The HST cutout (Figure 6) reveals just a bright nucleus surrounded by faint extended structure, the halflight radius being $3.2 \pm 0.5 \mathrm{kpc}$. The $K$-corrected mid-to-farinfrared spectral luminosities, $v L_{\nu}(5.8 \mu \mathrm{m})=3.6 \times 10^{8} L_{\odot}$ and $\nu L_{v}(24 \mu \mathrm{m})<1.0 \times 10^{9} L_{\odot}$, and the mid-infrared colors (Figure 7) show that the host is a star-forming galaxy.

Taken together, the radio luminosity, radio spectral index, optical morphology, and mid-to-far-infrared flux densities argue that ID 239 is a star-forming galaxy harboring a low-luminosity AGN.

ID 297. This source has a mean $1.4 \mathrm{GHz}$ flux density of $3.60 \pm 0.04 \mathrm{mJy}$. Huynh et al. (2012) measure a $5.5 \mathrm{GHz}$ flux density of $12.25 \mathrm{mJy}$, implying a spectral index of +0.89 . On the basis of this steep positive spectral index, Huynh et al. (2012) suggest that this is a part of a class of Gigahertz Peaked Spectrum sources, thought to be a young AGN. The redshift $z=0.605$ (Treister et al. 2009) implies a mean radio luminosity of log $L_{R}=31.5$ (cgs). ID 297 appears to be a stochastically varying source with no specific trend in its light curve (Figure 5). The radio position is consistent with a bright, unresolved $H S T$ source. On the basis of a departure from the radio-FIR correlation, Norris et al. classify this source as an AGN. The mid-infrared colors (Figure 7) and far-infrared spectral index (Table 3) are consistent with this identification.

ID 337. This source has a mean $1.4 \mathrm{GHz}$ flux density of $494 \pm 11 \mu \mathrm{Jy}$. From its radio light curve, ID 337 appears to have repeated outbursts roughly every 25 days. The flux density of this source in the Miller et al., Kellerman et al., Norris et al., and Afonso et al. catalogs is $439 \pm 8,524 \pm 14,380 \pm 16$, and $404 \pm 34 \mu \mathrm{Jy}$, respectively, with a spectral index of -0.2 between 1.4 and $4.8 \mathrm{GHz}$. The $5.5 \mathrm{GHz}$ flux density from Huynh et al. is $443 \pm 20 \mu \mathrm{Jy}$, for which we derive a spectral index of -0.08 . The photometric redshift of $z=0.734$ (Vanzella et al. 2008) implies a mean radio luminosity of $\log L_{R}=30.8$ (cgs).

The HST image (Figure 6) shows that the optical counterpart to the radio source is actually associated with the fainter (or more extincted) galaxy among a group of two closely separated galaxies. This has lead to some confusion over the correct optical identification. VLA observations carried out in 1999-2001 and 2007 (Kellerman et al. 2008 and Miller et al. 2008 , respectively) suggest that the radio counterpart is the fainter galaxy. ATCA observations carried out in 2003-2004 (Norris et al. 2006 and Afonso et al. 2006) suggest the bright galaxy as the optical counterpart. Our optical-radio frame tie summarized in Table 4 supports the fainter optical source as the likely radio counterpart. The spectral index and radio luminosity argue that ID 337 is a low-luminosity AGN. Afonso et al. (2006) state that the bright radio source is a luminous star-forming galaxy, possibly part of a merging system. Comparison of the $K$-corrected spectral luminosities, $v L_{\nu}(5.8 \mu \mathrm{m})=4.0 \times 10^{9} L_{\odot}$ and $\nu L_{v}(24 \mu \mathrm{m})<1.1 \times 10^{10} L_{\odot}$, with the Chary \& Elbaz (2001) SED templates and Desai et al. (2007), together with the mid-infrared colors (Figure 7) advocate the star-forming nature of the host galaxy.

ID 621. This source has a mean $1.4 \mathrm{GHz}$ flux density of $497 \pm 10 \mu \mathrm{Jy}$. The first epoch in its light curve reveals an initial brightening of the source followed by a decline and subsequent small-amplitude variations. The maximum flux density is close to $400 \%$ of the quiescent flux density of about $300 \mu \mathrm{Jy}$. The Miller et al., Kellerman et al., and Norris et al. catalogs list the flux density of this source as $494 \pm 10,565 \pm 17$, and $450 \pm 18 \mu \mathrm{Jy}$, respectively, with a spectral index of -0.1 between 1.4 and $4.8 \mathrm{GHz}$. The $5.5 \mathrm{GHz}$ flux density from Huynh et al. is $689 \pm 16 \mu \mathrm{Jy}$, for which we derive a spectral index of 0.24 . The photometric redshift of $z=1.107$ (Bonzini et al. 2012) implies a mean radio luminosity of $\log L_{R}=31.2$ (cgs).

The HST image reveals a bright nucleus surrounded by diffuse emission. The half-light radius is $\sim 3 \mathrm{kpc}$. The radio position is consistent with the nuclear source. Taken together, the radio luminosity, spectral index, and optical morphology argue that ID 621 is an AGN. Additionally, the $K$-corrected $5.8 \mu \mathrm{m}$ luminosity of $5.0 \times 10^{9} L_{\odot}$, the $24 \mu \mathrm{m}$ luminosity upper limit of $6.8 \times 10^{10} L_{\odot}$, and the mid-infrared colors (Figure 7) suggest that this is also a star-forming galaxy.

ID 628. This source has a mean $1.4 \mathrm{GHz}$ flux density of $1.34 \pm$ $0.02 \mathrm{mJy}$. The light curve indicates a steady increase of the quiescent emission to maximum flux density, followed by a steady decline. The Miller et al., Kellerman et al., and Norris et al. cata$\operatorname{logs}$ list the flux density of this source as $1.07 \pm 0.02,1.33 \pm 0.03$, and $0.90 \pm 0.02 \mu \mathrm{Jy}$. The $5.5 \mathrm{GHz}$ flux density from Huynh et al. is $0.78 \pm 0.02 \mu \mathrm{Jy}$, for which we derive a spectral index of -0.40 . The photometric redshift of $z=0.685$ (Norris et al. 2006) implies a mean radio luminosity of $\log L_{R}=31.2(\mathrm{cgs})$.

The HST image reveals a bright nucleus surrounded by disklike diffuse emission, the half-light radius being $\sim 10 \mathrm{kpc}$. The radio position is consistent with the nuclear source. On the basis of departure from the radio-FIR correlation, Norris et al. classify this source as an AGN, which is consistent with our flat radio spectral index and radio luminosity. Additionally, the $K$-corrected $5.8 \mu \mathrm{m}$ luminosity of $3.0 \times 10^{9} L_{\odot}$, the $24 \mu \mathrm{m}$ luminosity of $3.7 \times 10^{10} L_{\odot}$, and the mid-infrared colors (Figure 7) suggest that this is a normal star-forming or a starburst galaxy. Taken together, the radio and mid-to-farinfrared properties along with the optical morphology argue that ID 628 is an AGN embedded within a star-forming galaxy.

\section{TRANSIENT SEARCH}

For our transient search, we are interested in identifying those point sources which show up above the flux density limit for a short amount of time (corresponding to one or more epochs 
depending on the cadence), and remain below the limit in all other epochs. It is therefore important to reliably distinguish noise from real transients (which will determine how well we can reject false positives) and to avoid the rejection of transients as noise (reducing the number of true negatives). Thus, characterizing the effectiveness of source-finding algorithms in terms of reliability and completeness is crucial. Another motivation for characterizing source-finding algorithms is to find the optimum parameter values for use in transient searches.

In the following subsections, we begin by testing the efficacy of existing source-finding algorithms (Section 4.1). We then apply some of the better-performing algorithms to the E-CDFS epochs (Section 4.2).

\subsection{Efficacy of Source-finding Algorithms}

Recently, quantitative tests have been carried out on the reliability and completeness of source-finding algorithms (Huynh et al. 2011; Hancock et al. 2012). These studies found that of the many publicly available software packages, sfind and IMSAD in MIRIAD, Aegean, SExtractor, and Selavy, sfind and Aegean produce the most reliable catalogs.

The analysis of Huynh et al. (2011) and Hancock et al. (2012) was carried out on two simulated data sets. (i) The ASKAP simulation is a $4^{\circ} \times 4^{\circ}$ image of a full continuum observation with critically sampled beams in the $6 \mathrm{~km}$ ASKAP configuration. Its pixel scale is 2 .'75 and the rms noise is about $35 \mathrm{mJy}$, which varies across the field. Sixteen idealized beams one degree apart, spaced in a rectangular grid, mimic the effect of the phased-array feed. The image contains $\sim 7.7$ million sources having flux densities greater than $1 \mu \mathrm{Jy}$ from the $\mathrm{S}^{3}$ semiempirical extragalactic simulation (Wilman et al. 2010). (ii) For the Hancock et al. simulation, a sky image was created as a $4801^{2}$ pixel image $8^{\circ}$ across with $6^{\prime \prime}$ pixels sampling a $30^{\prime \prime}$ beam and an rms noise of $25 \mu \mathrm{Jy}$. Sources were injected at random positions with angular sizes (with random position angles) from $0^{\prime \prime}$ to $52^{\prime \prime}$ and with source number counts distributed with peak flux densities as $N(S) \propto S^{-2.3}$ such that 15,000 sources having fluxes densities $>1 \sigma$ are present in the image.

With our E-CDFS data set, we are able to carry out a similar analysis using real data with all its attendant residual calibration and imaging errors. A comparison of the real and simulated data could be informative. While simulations are useful in determining which source-finding algorithm works best in general, they do not explore the parameter space of the algorithm thoroughly. Hence, they may not provide optimum parameter values for a transient search on a specific data set. The deep field of the E-CDFS is well suited for this comparison. The $4096^{2}$ pixel image is $34^{\prime}$ across with $00^{\prime} .5$ pixels sampling a synthesized beam of $2^{\prime \prime} .8 \times 1^{\prime \prime} .6$ (position angle $\simeq 0^{\circ}$ ) and an rms noise 7.4 $\mu \mathrm{Jy}$. As outlined in Section 3, great care was taken in constructing the DR2 source catalog so we can be assured of its completeness and reliability (see also Figure 2).

In what follows, we will use the DR2 catalog and the deep E-CDFS image to test various source-finding algorithms for completeness and reliability for different input parameters. We use the terms "real sources" and "false sources" for those sources that are present in the DR2 catalog and those that are not, respectively. ${ }^{11}$ Whether a source detected by a source-finding

\footnotetext{
11 Even though the DR2 catalog was constructed with great care, it is likely that $\sim 1$ genuine source was missed and a handful of spurious sources added (perhaps not truly spurious, but SAD sources at $4 \sigma$ bumped up to $5 \sigma$ by the JMFIT task in AIPS). Recall that according to the Eddington bias, more sub- $5 \sigma$ sources get bumped up than $5 \sigma$ get bumped down.
}

algorithm has a counterpart in the DR2 catalog is determined by searching for DR2 sources within $1^{\prime \prime}$ of the source position. Following Huynh et al. (2011), we define two additional terms: "completeness," as the fraction of real sources detected by a source-finding algorithm, and "reliability," as the fraction of detected sources which are real. Note that in Figures 8, 9, and 12, we plot the completeness and reliability within contiguous $\mathrm{S} / \mathrm{N}$ bins, unlike Huynh et al. (2011) and Hancock et al. (2012) where, for a given $\mathrm{S} / \mathrm{N}$, the plots represent the completeness and reliability for sources greater than or equal to that $S / N$. For source-finding algorithms employing a probabilistic approach of drawing a pixel from the background and thus calculating the false-detection rate (FDR), FDR + Reliability $=100 \%$.

$$
\text { 4.1.1. sfind (MIRIAD) }
$$

In its default mode, $\mathrm{sf}$ ind ${ }^{12}$ incorporates a statistically robust method for detecting source pixels, called "false discovery rate," or FDR. In the FDR algorithm, detected sources are drawn from a distribution of pixels with a robustly known chance of being falsely drawn from the background. Contiguous, monotonically decreasing adjacent pixels from the FDR-selected ones, are used for fitting two-dimensional (2D) elliptical Gaussians to the sources. Thus, the fraction of expected false sources is more reliably determined than in sigma-clipping criteria (see methods below). Details of the FDR method can be found in Hopkins et al. (2001). The run time for sfind searching for sources down to $5 \sigma$ in the DR2 image is about $20 \mathrm{~s}$. For all the tests carried out on sfind, MIRIAD version 4.2.3 (optimized for CARMA; CVS Revision 1.11, 2011 April 26) was used.

Here, we explore the completeness and reliability of sfind by tweaking the two relevant parameters: (i) alpha( $\alpha$ ), the percentage of probable background pixels that can be accepted in the analysis; and (ii) rmsbox, the size of the smoothing box used for estimating the background and the standard deviation of the image. Table 5 lists the parameter values tested.

In general, the completeness of sfind increases and its reliability decreases (Table 5) with increasing $\alpha$, as expected for an FDR algorithm. Also, both of these quantities increase with rmsbox. The rise in completeness and reliability is precipitous (between 5\% and 20\%) as rmsbox is increased from 5 to 10 beamwidths, after which it flattens off. There is a slight decrease in the reliability as rmsbox is increased from 20 to 50 beamwidths. Ten beamwidths can then be interpreted as the minimum box size for determining the background rms noise without significant contribution from the sources themselves, whereas 50 beamwidths would correspond to the size where the calculated rms starts deviating significantly from the true local rms. However, in the case of bright sources in the field, these rmsbox limits might be somewhat larger. Completeness and reliability are $\sim 88 \%$ for $\alpha=10$ and rmsbox $=20$, but better reliability (by a few percent) can be obtained at an equal expense of completeness by using $\alpha=5$ or 2 . Further decrease in $\alpha$ substantially decreases the completeness without any significant improvement in the reliability. Thus, we determine the optimum values for the input parameters to be $5 \leqslant \alpha \leqslant 10$ and $10 \leqslant$ rmsbox $\leqslant 50$ beamwidths. For the FDR algorithm, we expect the reliability to be $1-\alpha$. However, sf ind reliability is less than this expected value by a few percent due to the acceptance of sidelobes of bright sources and the fitting of extended sources with multiple elongated and overlapping (unphysical) components. To some extent, the acceptance of

\footnotetext{
12 www.atnf.csiro.au/computing/software/miriad/doc/sfind.html
} 

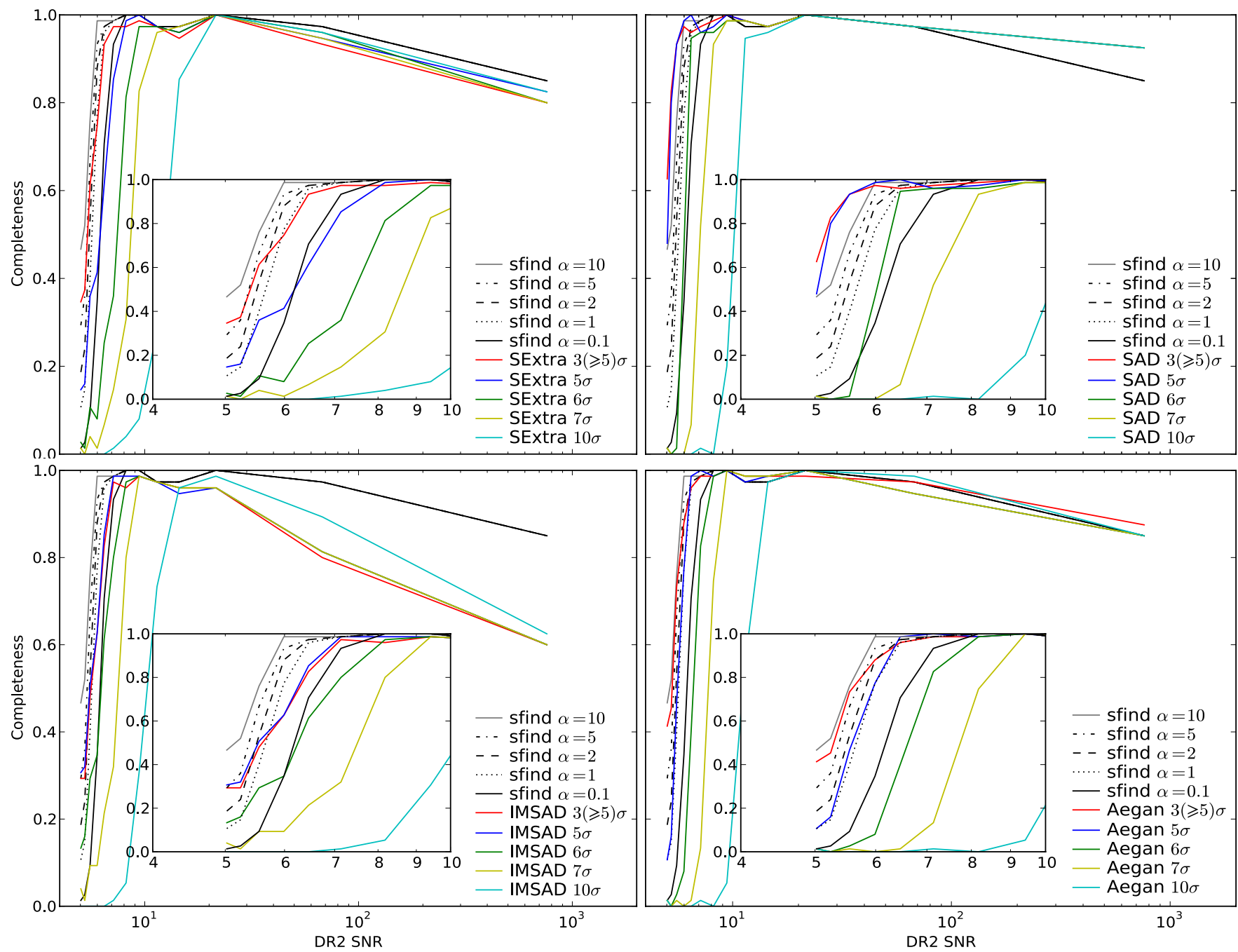

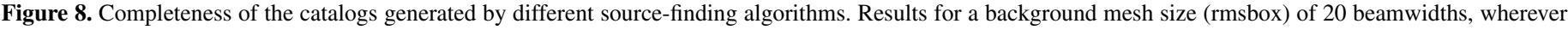

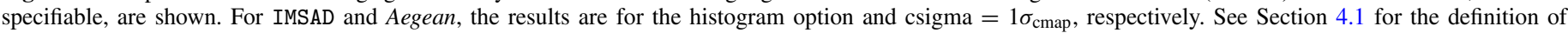

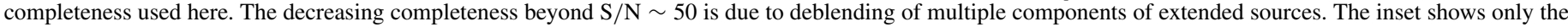

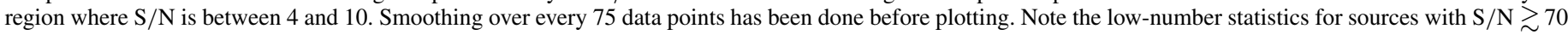
as implied by Figure 2 .

(A color version of this figure is available in the online journal.)

these false sources can be reduced by choosing a lower value of $\alpha$. Some examples of the components fit by sfind to different kinds of sources in different environments in the DR2 image are shown in Figure 6. In general, compared to other source-finding algorithms, except for SAD, sfind has a better reliability for a given completeness, and vice versa (Tables 5-9; see also Section 4.1.3 for a note on the efficacy of SAD). These findings are consistent with the tests carried out on simulated images (Huynh et al. 2011). In the ASKAP simulation, the reliability of the sfind catalog is rather flat with respect to $\alpha$, whereas for the Hancock et al. simulation, it decreases by several percent as $\alpha$ increases from 0.1 to 10 (Huynh et al. 2011). In the latter, rmsbox $=20$ is found to give slightly better reliability than rmsbox $=20$. For the $\sim 3 \sigma-10 \sigma$ sources in these simulations, the completeness for $\alpha=5$ is greater than that for $\alpha=0.1$ by $5 \%-10 \%$. They do not explore the completeness for $\alpha=10$ and rmsbox other than 10 beamwidths.

Figure 8 shows the completeness of the sfind catalog as a function of the $\mathrm{S} / \mathrm{N}$ of the detected sources for different values of the input parameter $\alpha$. The reduction in completeness beyond $\mathrm{S} / \mathrm{N} \sim 20$ is due to missed or badly fit components of extended sources. The completeness for optimum values of the input parameters is shown in the upper panel of Figure 9, which shows that sfind reaches $100 \%$ completeness at a much lower $\mathrm{S} / \mathrm{N}$ than other algorithms. The lower panel of Figure 9 shows the reliability. Curiously, there is a dip in the reliability between $\mathrm{S} /$ Ns of 6 and 10. Point sources strewn across the DR2 image but primarily located near its edges (in regions of increased rms; see the lower panel of Figure 10, for example), which are absent in the DR2 catalog and detected by sfind, are responsible for this reduced reliability. Some of these sources are rejected from the sfind catalog when a lower value of $\alpha$ or rmsbox is used, indicating that they are either not genuine sources on the sky or are sources at a lower $\mathrm{S} / \mathrm{N}$. Indeed, other algorithms find some of these sources to be at a much reduced $\mathrm{S} / \mathrm{N}$ between 3 and 5. The results of Hancock et al. (2012) also show a dip in the sfind reliability, although centered on $\mathrm{S} / \mathrm{N} \sim 15$. Figure 11 compares the $\mathrm{S} / \mathrm{N}$ of sources detected by sfind with those of the counterparts from the DR2 catalog. The largely increased $\mathrm{S} / \mathrm{N}$ reported for sources at or below $6 \sigma$ in the DR2 catalog and the largely reduced $\mathrm{S} / \mathrm{N}$ for sources above $\sim 100 \sigma$ is anomalous. Although for $\mathrm{S} / \mathrm{N}$ above 100 the discrepancy is likely to be 

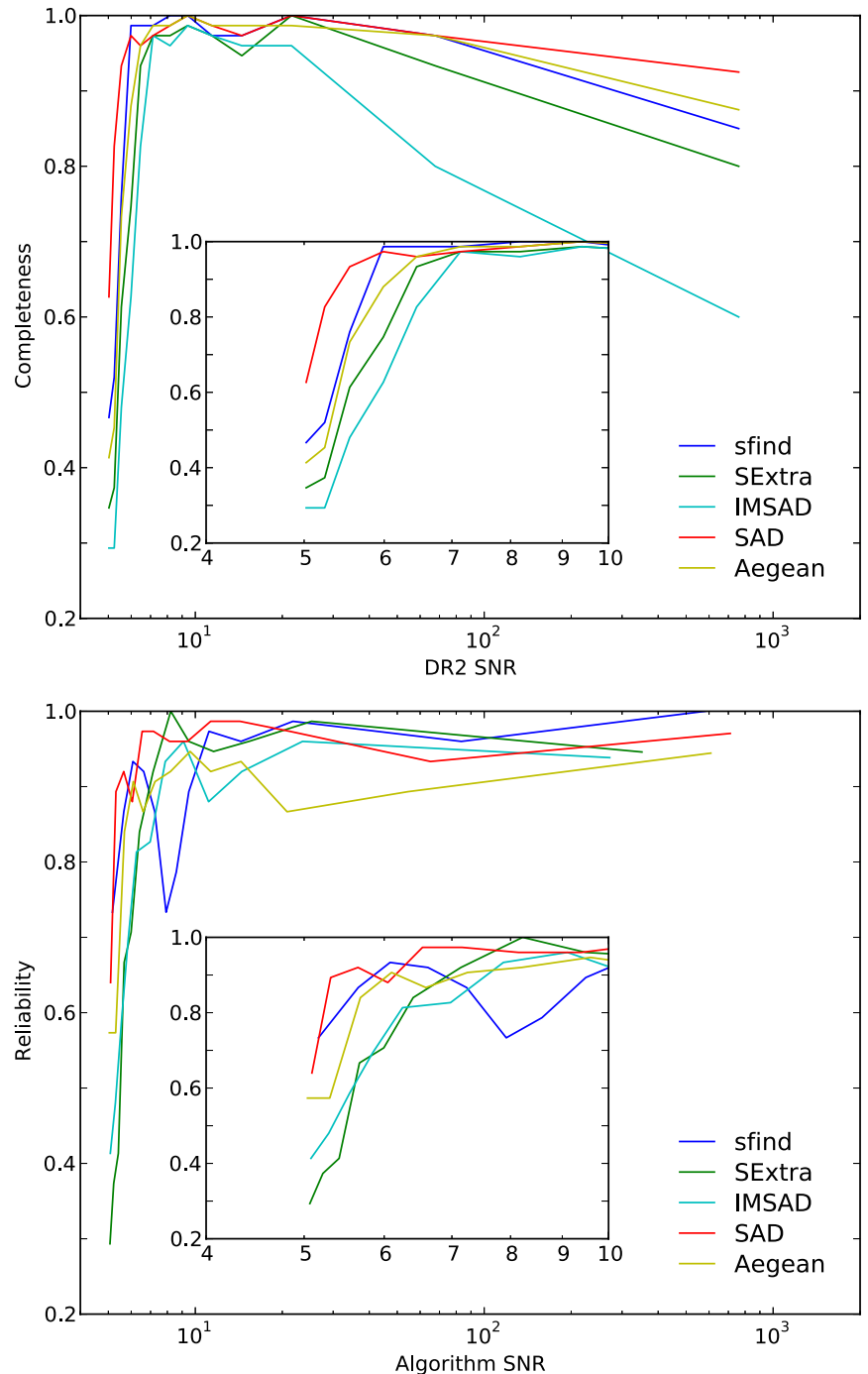

Figure 9. Completeness (top) and reliability (bottom) of the catalogs generated by different source-finding algorithms. See Section 4.1 for the definitions of completeness and reliability used in this work. Results for a background meshsize (rmsbox) of 20 beamwidths are shown. For IMSAD, these results are for the histogram option. For sfind, we have used $\alpha=10$, whereas for SExtractor, SAD, IMSAD, and Aegean, we used a detection threshold of $3 \sigma$. Only those sources which are $\geqslant 5 \sigma$ have then been selected from the respective catalogs prior to comparison with the DR2 catalog. The inset shows only the region where $\mathrm{S} / \mathrm{N}$ is between 4 and 10. Smoothing over all 75 data points has been done before plotting. Note the low-number statistics for sources with $S / N \gtrsim 70$ as implied by Figure 2.

(A color version of this figure is available in the online journal.)

due to overlapping components fit to extended sources, all the above observations indicate that the $\mathrm{S} / \mathrm{N}$ reported by sfind is somewhat different from that reported by other algorithms. Sources with $6<\mathrm{S} / \mathrm{N}<10$ in the sfind catalog clearly have overestimated (upto 100\%) peak flux densities and different dimensions than what would be expected from inspecting the cutouts of these sources. Thus, fitting of sources rather than differently reported rms is the cause of the S/N discrepancy, at least at these low S/Ns. The solution of this issue is possibly in correctly setting the fdrpeak and psfsize input parameters which allow reasonable measurements of sources close to the threshold. Since there are not many sources in our data having $\mathrm{S} / \mathrm{N} \gtrsim 70$ (Figure 2), the suggested trend in completeness, reliability, and measured $\mathrm{S} / \mathrm{N}$ in this domain should be treated with caution.

\subsubsection{SExtractor}

SExtractor ${ }^{13}$ is a source-finding program widely used in optical astronomy, and is particularly oriented toward the reduction of large-scale galaxy-survey data as well as sparsely to moderately crowded stellar fields. It analyzes the image in two passes such that in the first pass, a background map is made, and in the second, background subtraction, filtering, and thresholding is done on-the-fly. Detected sources are then deblended and CLEANed before performing photometry. The run time for SExtractor searching for sources down to $5 \sigma$ in the DR2 image is about $2 \mathrm{~s}$. For all the tests carried out here, the latest release of SExtractor (version 2.8.6, 2009 April 9) has been used.

We adopted a strategy of searching for sources down to $\mathrm{N \sigma}$ $(N=3,5,6,7,10$, as set using the DETECT_THRESH parameter in SExtractor) and then selecting the ones $\geqslant 5 \sigma$ prior to the comparison with the DR2 sources. Two input parameters (apart from possibly DEBLEND_NTHRESH, BACK_FILTTHRESH, and CLEAN_PARAM, which we have not tested) are expected to affect the completeness and reliability of the SExtractor catalog. First, the mesh size, which determines the size of the box used for background rms estimation, is specified by BACK SIZE, and second, the size of the median filter (BACK_FILTERSIZE) is applied to the background grid used for smoothing large artifacts in the image.

In Table 6, we list the completeness and reliability as a function of these parameters. Note that in this table, in order to reflect the true completeness for the specified detection threshold, the completeness values of the SExtractor catalog for the $6 \sigma, 7 \sigma$, and $10 \sigma$ thresholds have been normalized by the percentage of sources in the DR2 catalog that are beyond these thresholds, respectively. Thus, the completeness at any threshold is expected to be $100 \%$ if all the DR2 sources beyond that threshold are detected by SExtractor. The general trend observed is that, with increasing detection threshold, the completeness of SExtractor is fairly steady, but its reliability increases. However, for $5 \sigma$ and $6 \sigma$ detection thresholds, the completeness is rather low $(\sim 75 \%$ or lower). Choosing higher detection thresholds such as $7 \sigma-10 \sigma$ does not seem to improve the completeness substantially. This is due to several factors through which the source finding in SExtractor seems to be different from the conventionally used algorithms in radio astronomy. First, for any given source the reported peak flux density decreases as the detection threshold is raised, although there is not much of a change in the reported rms. Hence, the $\mathrm{S} / \mathrm{N}$ of sources close to the detection threshold decreases so as to be rejected by SExtractor. Choosing a $3 \sigma$ threshold usually gives the correct peak flux density. This is also the reason why searching down to $3 \sigma$ and selecting sources at the desired higher threshold increases the completeness (although with the side effect of reduced reliability). The explanation for such an effect is hinted at by the fact that the dimensions of the fitted sources decrease (this effect is quite significant for sources with $\mathrm{S} / \mathrm{N}<10$ ) with increasing detection threshold, implying that the number of source pixels considered in the fitting process depends on the threshold. Second, several DR2 sources that have $\mathrm{S} / \mathrm{N}<10$ and are detected by other source-finding algorithms (excepting IMSAD) are not detected at all by SExtractor, not even at a reduced $\mathrm{S} / \mathrm{N}$. This might be due to the differences in the fitting process rather than a discrepant rms. Finally, for some extended sources, the positions of the components reported by SExtractor are at least a few synthesized beams away from the

\footnotetext{
13 www.astromatic.net/software/sextractor
} 

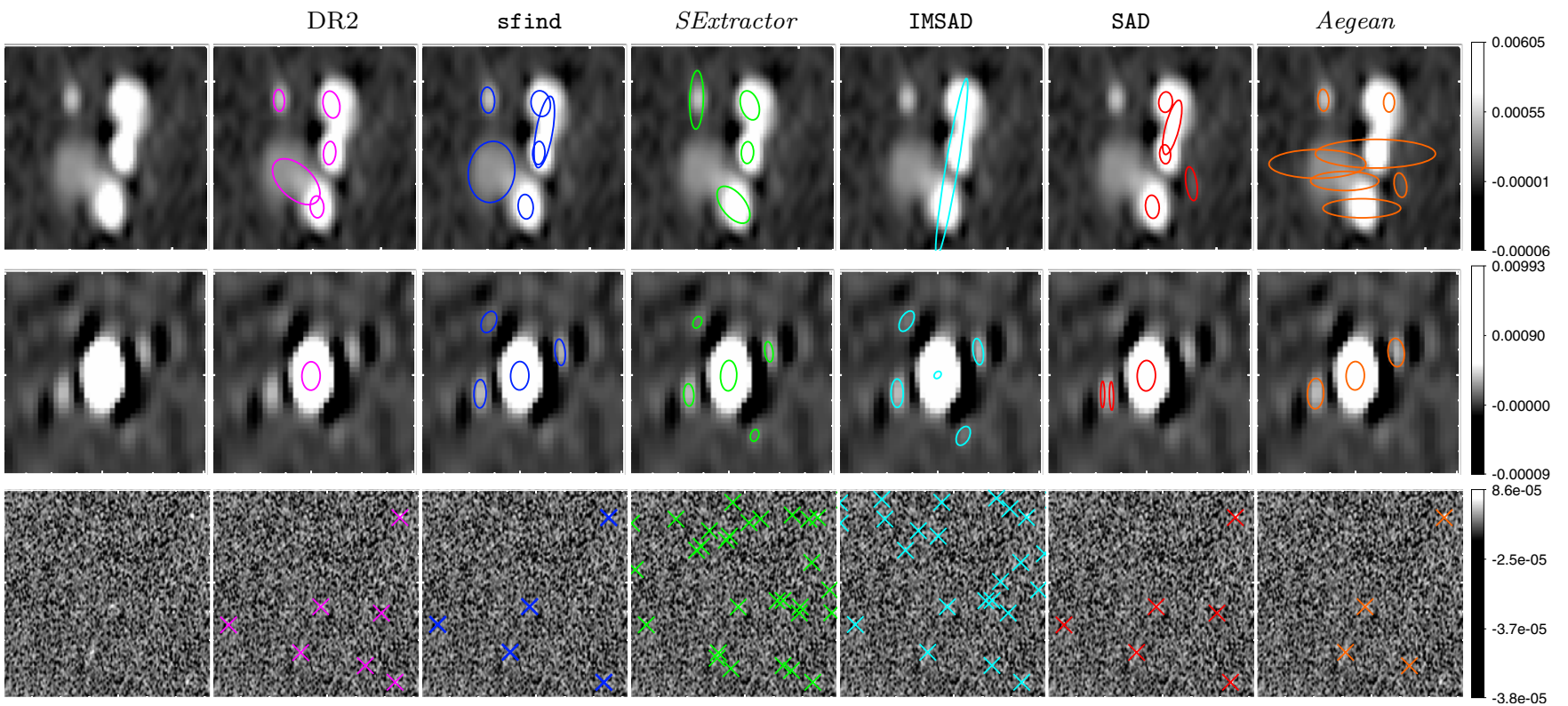

Figure 10. Examples of $5 \sigma$ and brighter sources detected by various source-finding algorithms in the DR2 image. Top panel: blended components (30" cutouts centered on $\left.03^{\mathrm{h}} 32^{\mathrm{m}} 32^{\mathrm{s}} \cdot 2,-28^{\circ} 03^{\prime} 09^{\prime \prime} 4\right)$, middle panel: source with sidelobes $\left(20^{\prime \prime}\right.$ cutouts centered on $03^{\mathrm{h}} 32^{\mathrm{m}} 06^{\mathrm{s}} .1,-27^{\circ} 32^{\prime} 35^{\prime \prime}$. 8$)$, and bottom panel: region with a relatively large rms $(12 \mu \mathrm{Jy})$ at the corner of the image $\left(3^{\prime}\right.$ cutouts centered on $\left.03^{\mathrm{h}} 31^{\mathrm{m}} 19^{\mathrm{s}} .4,-27^{\circ} 32^{\prime} 55^{\prime \prime} \cdot 6\right)$. The logarithmic flux density scale shown in each panel has units of Jy beam ${ }^{-1}$. The ellipses have major and minor axes and position angles according to the parameters reported by the respective algorithms.

(A color version of this figure is available in the online journal.)

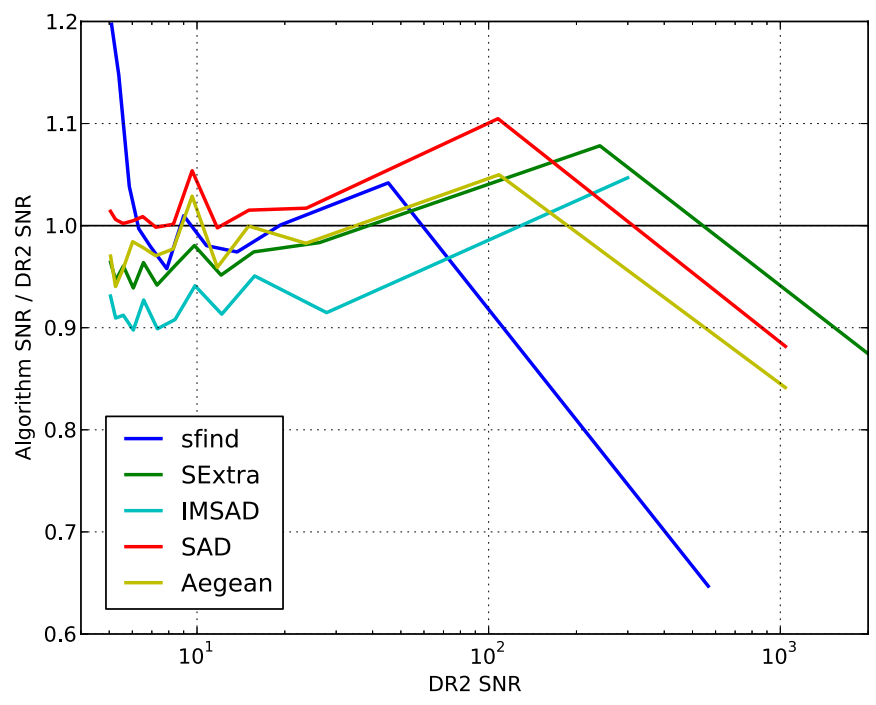

Figure 11. $\mathrm{S} / \mathrm{N}$ of sources found by different source-finding algorithms relative to their $\mathrm{S} / \mathrm{N}$ in the DR2 catalog. Smoothing over all 75 data points has been done before plotting. Note the low-number statistics for sources with $\mathrm{S} / \mathrm{N} \gtrsim 70$ as implied by Figure 2 .

(A color version of this figure is available in the online journal.)

positions given in the DR2 catalog; the latter almost always agree with the positions reported by other source-finding algorithms (again, with the exception of IMSAD; see Section 4.1.4 for anomalies associated with IMSAD). The completeness peaks at a background mesh size of 5 beamwidths used with $3 \times$ smoothing, whereas reliability appears to increase steadily or remain constant with increasing mesh size. BACK_FILTERSIZE is not seen to change either the completeness or reliability much. SExtractor does not seem to achieve a high completeness and reliability $(>85 \%)$ simultaneously for any given set of values for the input parameters. For a reliability of $>90 \%$, we see that completeness $<80 \%$. Beyond a detection threshold of $6 \sigma$, the

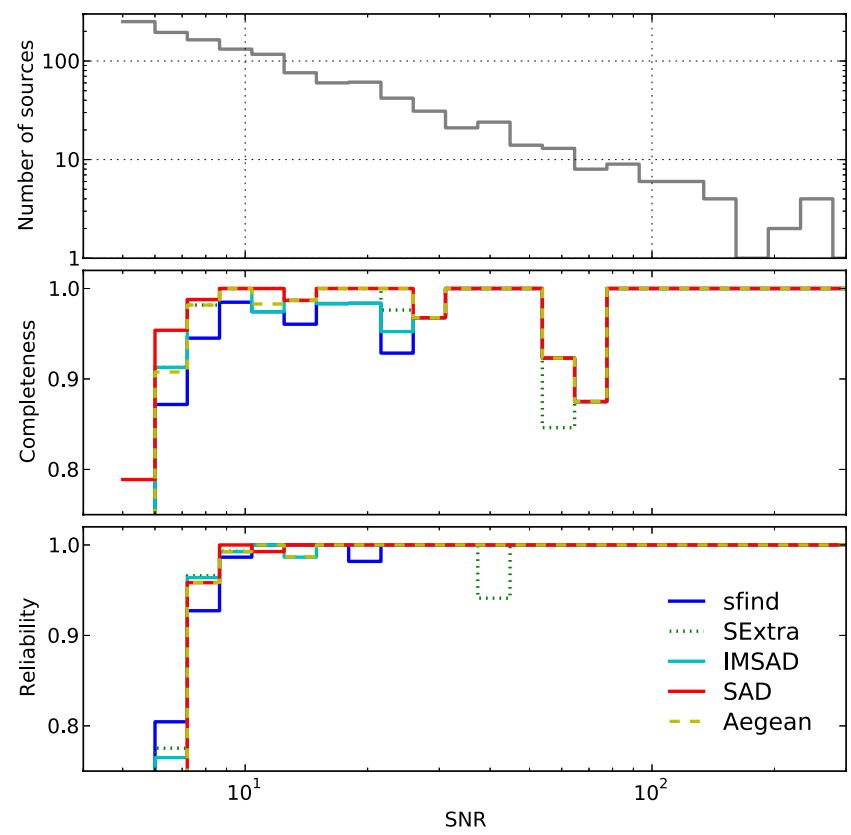

Figure 12. Results of the completeness (middle) and reliability (bottom) tests on the Hancock et al. simulated image. A matching radius of $15^{\prime \prime}$ was used to find counterparts. Input parameters to the source-finding algorithms are same as those given in Figure 9. Here, the completeness and reliability within each S/N bin are plotted (see Section 4.1), which makes these diagrams different from the ones given in Hancock et al. (2012). For reference, the histogram of the simulated sources is also shown (top). The completeness at $\mathrm{S} / \mathrm{N} \sim 70$ dropping below $90 \%$ is a result of highly blended sources.

(A color version of this figure is available in the online journal.)

reliability is quite good, yet less than $100 \%$ owing primarily to the acceptance of sidelobes and the differently handled fitting of extended sources. Nevertheless, the highest reliability for SExtractor is better than the catalogs of other algorithms (except, maybe SAD), and hence it is best for cases where reliability is strongly favored over completeness. In such a case, we 
Table 5

Completeness and Reliability of sfind Catalog

\begin{tabular}{|c|c|c|c|c|c|}
\hline \multirow{2}{*}{$\begin{array}{l}\text { rmsbox } \\
\text { (beamwidths) }\end{array}$} & \multicolumn{5}{|c|}{$\alpha$} \\
\hline & 10 & 5 & 2 & 1 & 0.1 \\
\hline \multicolumn{6}{|c|}{ Completeness } \\
\hline 5 & $80.9 \%$ & $77.8 \%$ & $74.3 \%$ & $72.4 \%$ & $61.9 \%$ \\
\hline 10 & $85.7 \%$ & $83.7 \%$ & $79.1 \%$ & $75.9 \%$ & $65.5 \%$ \\
\hline 20 & $88.4 \%$ & $84.5 \%$ & $81.2 \%$ & $77.8 \%$ & $67.8 \%$ \\
\hline 50 & $89.1 \%$ & $85.7 \%$ & $82.1 \%$ & $78.4 \%$ & $68.0 \%$ \\
\hline \multicolumn{6}{|c|}{ Reliability } \\
\hline 5 & $78.8 \%$ & $84.9 \%$ & $90.6 \%$ & $93.1 \%$ & $96.4 \%$ \\
\hline 10 & $86.8 \%$ & $91.6 \%$ & $95.2 \%$ & $95.6 \%$ & $97.4 \%$ \\
\hline 20 & $88.7 \%$ & $92.5 \%$ & $95.3 \%$ & $96.0 \%$ & $96.4 \%$ \\
\hline 50 & $87.5 \%$ & $91.4 \%$ & $93.8 \%$ & $94.8 \%$ & $95.3 \%$ \\
\hline
\end{tabular}

Notes. Only $\geqslant 5 \sigma$ sources have been selected from the sfind catalogs prior to comparison with the DR2 catalog.

Table 6

Completeness and Reliability of SExtractor Catalog

\begin{tabular}{|c|c|c|c|c|c|}
\hline \multirow{2}{*}{$\begin{array}{l}\text { BACK_SIZE } \\
\text { (beamwidths) }\end{array}$} & \multicolumn{5}{|c|}{ DETECT_THRESH } \\
\hline & $3(\geqslant 5)$ & 5 & 6 & 7 & 10 \\
\hline \multicolumn{6}{|c|}{ Completeness* } \\
\hline 5 & $82.2 \%$ & $72.1 \%$ & $76.8 \%$ & $79.1 \%$ & $77.9 \%$ \\
\hline $5+3 \times$ smoothing & $83.4 \%$ & $73.7 \%$ & $78.7 \%$ & $80.0 \%$ & $79.0 \%$ \\
\hline $5+10 \times$ smoothing & $83.3 \%$ & $73.0 \%$ & $78.4 \%$ & $80.0 \%$ & $79.0 \%$ \\
\hline 10 & $81.7 \%$ & $71.4 \%$ & $76.9 \%$ & $78.7 \%$ & $78.2 \%$ \\
\hline $10+3 \times$ smoothing & $82.1 \%$ & $71.1 \%$ & $76.7 \%$ & $79.1 \%$ & $77.6 \%$ \\
\hline $10+10 \times$ smoothing & $81.7 \%$ & $71.2 \%$ & $76.4 \%$ & $78.6 \%$ & $77.6 \%$ \\
\hline 20 & $81.6 \%$ & $70.6 \%$ & $75.9 \%$ & $78.6 \%$ & $77.6 \%$ \\
\hline 50 & $81.3 \%$ & $70.2 \%$ & $75.3 \%$ & $78.0 \%$ & $77.3 \%$ \\
\hline \multicolumn{6}{|c|}{ Reliability } \\
\hline 5 & $70.4 \%$ & $87.5 \%$ & $95.1 \%$ & $96.3 \%$ & $97.0 \%$ \\
\hline $5+3 \times$ smoothing & $72.4 \%$ & $89.0 \%$ & $96.1 \%$ & $97.2 \%$ & $97.4 \%$ \\
\hline $5+10 \times$ smoothing & $72.2 \%$ & $89.5 \%$ & $96.1 \%$ & $97.2 \%$ & $97.4 \%$ \\
\hline 10 & $75.2 \%$ & $90.8 \%$ & $96.2 \%$ & $96.5 \%$ & $97.4 \%$ \\
\hline $10+3 \times$ smoothing & $75.8 \%$ & $90.1 \%$ & $96.2 \%$ & $97.0 \%$ & $97.4 \%$ \\
\hline $10+10 \times$ smoothing & $75.6 \%$ & $90.2 \%$ & $96.2 \%$ & $96.9 \%$ & $97.4 \%$ \\
\hline 20 & $77.0 \%$ & $90.3 \%$ & $96.4 \%$ & $96.9 \%$ & $97.4 \%$ \\
\hline 50 & $78.1 \%$ & $91.1 \%$ & $96.5 \%$ & $96.9 \%$ & $97.4 \%$ \\
\hline
\end{tabular}

Notes. ${ }^{*}$ The fraction of sources in the DR2 catalog which are $\geqslant 6 \sigma, 7 \sigma$, and $10 \sigma$ are $73.0 \%, 58.1 \%$, and $37.7 \%$, respectively. Completeness for these detection thresholds has been normalized accordingly. A detection threshold of $3(\geqslant 5)$ implies a search down to $3 \sigma$ followed by the selection of only those source that are greater than $5 \sigma$.

recommend setting the detection threshold to about $10 \sigma$, along with a large enough value for BACK SIZE. Tests on SExtractor with the ASKAP and Hancock et al. simulations (Huynh et al. 2011) suggest that the reliability is almost constant for mesh sizes between 10 and 100 beamwidths, but increases with the detection threshold. They find that the completeness generally decreases or remains constant as mesh size increases, but its change with respect to detection threshold is not explored. Our results are thus broadly consistent with the tests on the simulated images.

The upper-left panel of Figure 8 shows the completeness of SExtractor sources as a function of their $\mathrm{S} / \mathrm{N}$ in the DR2 catalog for a mesh size of 20 beamwidths and different values of the detection threshold. The completeness for optimum input
Table 7

Completeness and Reliability of SAD Catalog

\begin{tabular}{|c|c|c|c|c|c|}
\hline \multirow{2}{*}{$\begin{array}{l}\text { IMSIZE } \\
\text { (beamwidths) }\end{array}$} & \multicolumn{5}{|c|}{ CPARM } \\
\hline & $3(\geqslant 5)$ & 5 & 6 & 7 & 10 \\
\hline \multicolumn{6}{|c|}{ Completeness* } \\
\hline 5 & $77.8 \%$ & $75.5 \%$ & $80.0 \%$ & $83.7 \%$ & $78.4 \%$ \\
\hline 10 & $89.9 \%$ & $88.7 \%$ & $93.1 \%$ & $92.1 \%$ & $93.4 \%$ \\
\hline 20 & $93.4 \%$ & $92.2 \%$ & $95.9 \%$ & $95.4 \%$ & $97.1 \%$ \\
\hline 50 & $95.9 \%$ & $94.8 \%$ & $96.5 \%$ & $95.8 \%$ & $97.1 \%$ \\
\hline actnoise $^{a}$ & $87.4 \%$ & $86.7 \%$ & $95.2 \%$ & $96.7 \%$ & $95.7 \%$ \\
\hline \multicolumn{6}{|c|}{ Reliability } \\
\hline 5 & $88.1 \%$ & $89.0 \%$ & $97.3 \%$ & $99.1 \%$ & $99.3 \%$ \\
\hline 10 & $91.2 \%$ & $93.0 \%$ & $97.4 \%$ & $97.4 \%$ & $96.6 \%$ \\
\hline 20 & $92.5 \%$ & $93.9 \%$ & $96.7 \%$ & $96.9 \%$ & $97.0 \%$ \\
\hline 50 & $85.1 \%$ & $87.3 \%$ & $88.1 \%$ & $88.6 \%$ & $92.1 \%$ \\
\hline actnoise $^{\mathrm{a}}$ & $58.2 \%$ & $60.2 \%$ & $86.6 \%$ & $94.6 \%$ & $96.4 \%$ \\
\hline
\end{tabular}

Note. ${ }^{a}$ Search using the actnoise keyword in the FITS header $(=7.465 \mathrm{E}$ $06 \mathrm{JY} / \mathrm{BM})$.

* The fraction of sources in the DR2 catalog which are $\geqslant 6 \sigma, 7 \sigma$, and $10 \sigma$ are $73.0 \%, 58.1 \%$, and $37.7 \%$, respectively. Completeness for these detection thresholds has been normalized accordingly. A detection threshold of $3(\geqslant 5)$ implies a search down to $3 \sigma$ followed by the selection of only those source that are greater than $5 \sigma$.

Table 8

Completeness and Reliability of IMSAD Catalog

\begin{tabular}{lccccc}
\hline \hline options & \multicolumn{5}{c}{ clip } \\
\cline { 2 - 6 } & $3(\geqslant 5)$ & 5 & 6 & 7 & 10 \\
\hline \multicolumn{5}{c}{ Completeness* } \\
\hline noplt $^{\mathrm{a}}$ & $75.7 \%$ & $73.9 \%$ & $78.1 \%$ & $84.2 \%$ & $79.3 \%$ \\
noplt, hist $^{7}$ & $75.4 \%$ & $76.5 \%$ & $91.1 \%$ & $90.5 \%$ & $90.6 \%$ \\
\hline noplt & Reliability \\
noplt, hist & $77.4 \%$ & $83.7 \%$ & $95.3 \%$ & $96.5 \%$ & $96.8 \%$ \\
\hline
\end{tabular}

Note. ${ }^{\text {a }}$ Clipping level is manually entered as the appropriate multiple of the background rms chosen to be $8 \mu \mathrm{Jy}$.

* The fraction of sources in the DR2 catalog which are $\geqslant 6 \sigma, 7 \sigma$, and $10 \sigma$ are $73.0 \%, 58.1 \%$, and $37.7 \%$, respectively. Completeness for these detection thresholds has been normalized accordingly. A detection threshold of $3(\geqslant 5)$ implies a search down to $3 \sigma$ followed by the selection of only those source that are greater than $5 \sigma$.

Table 9

Completeness and Reliability of Aegean Catalog

\begin{tabular}{|c|c|c|c|c|c|}
\hline \multirow{2}{*}{$\begin{array}{l}\text { csigma } \\
\text { (cmap rms) }\end{array}$} & \multicolumn{5}{|c|}{ innerclip = outerclip } \\
\hline & $3(\geqslant 5)$ & 5 & 6 & 7 & 10 \\
\hline \multicolumn{6}{|c|}{ Completeness* } \\
\hline 0.5 & $86.1 \%$ & $78.7 \%$ & $83.8 \%$ & $86.1 \%$ & $85.8 \%$ \\
\hline 1.0 & $86.3 \%$ & $78.5 \%$ & $84.2 \%$ & $86.4 \%$ & $85.8 \%$ \\
\hline 2.0 & $88.2 \%$ & $78.5 \%$ & $84.5 \%$ & $86.8 \%$ & $85.5 \%$ \\
\hline \multicolumn{6}{|c|}{ Reliability } \\
\hline 0.5 & $86.2 \%$ & $91.6 \%$ & $91.6 \%$ & $92.1 \%$ & $91.7 \%$ \\
\hline 1.0 & $84.9 \%$ & $90.6 \%$ & $90.5 \%$ & $90.5 \%$ & $91.1 \%$ \\
\hline 2.0 & $83.4 \%$ & $91.0 \%$ & $91.2 \%$ & $91.4 \%$ & $89.9 \%$ \\
\hline
\end{tabular}

Notes. ${ }^{*}$ The fraction of sources in the DR2 catalog which are $\geqslant 6 \sigma, 7 \sigma$, and $10 \sigma$ are $73.0 \%, 58.1 \%$, and $37.7 \%$, respectively. Completeness for these detection thresholds has been normalized accordingly. A detection threshold of $3(\geqslant 5)$ implies a search down to $3 \sigma$ followed by the selection of only those source that are greater than $5 \sigma$. 
parameter values is shown in the upper panel of Figure 9. SExtractor approaches $100 \%$ completeness faster than IMSAD, but slower than the other algorithms. This is possibly related to the approach used for determining source pixels. The decrease in completeness beyond $\mathrm{S} / \mathrm{N} \sim 20$ can be attributed to the extended-source components being reported differently than other algorithms, as mentioned above. The lower panel of Figure 9, which shows the SExtractor reliability in relation to other source-finding algorithms, reveals that this algorithm gives quite unreliable results for $\mathrm{S} / \mathrm{N} \lesssim 10$. These low-S/N false sources are all point sources located near the edges of the DR2 image where the rms is somewhat large $(12 \mu \mathrm{Jy})$, but the rms reported by SExtractor is quite small $(7-8 \mu \mathrm{Jy})$. This scenario is presented in the image cutouts in Figure 10, which also illustrate the ability of SExtractor to find sources with different morphologies located in different environments. Figure 11 shows the $\mathrm{S} / \mathrm{N}$ of sources detected by SExtractor, in comparison with the corresponding sources in the DR2 catalog. For $\mathrm{S} / \mathrm{N} \leqslant 30$, the peak flux density of the SExtractor sources sfind, SExtractor is generally less than that of the DR2 catalog sources, suggesting that the difference in the calculated peak flux density (and to a smaller extent, the associated uncertainty) is responsible for the observed departure of the SExtractor $\mathrm{S} / \mathrm{N}$ from the DR2 S/N.

$$
\text { 4.1.3. SAD (AIPS) }
$$

The "Search and Destroy" $\left(\mathrm{SAD}^{14}\right)$ algorithm finds all the pixels above a specified threshold (typically a multiple of the rms noise, which is assumed to be Gaussian) in the image, and merges contiguous pixels above the threshold into islands. The strength and size of each island is then estimated, followed by least-squares Gaussian fitting of each island (if rms residual is too high, then multiple Gaussian fits may be applied). However, note that Gaussian statistics may not be a good model for the distribution of values in pixels well above zero flux density on account of thermal noise, and calibration and imaging artifacts (Cotton \& Peters 2011). The run time of SAD searching for sources down to $5 \sigma$ in the DR2 image is about 2 minutes. Additionally, to prepare the background rms image, the task RMSD takes $\sim 15$ minutes. For all the tests carried out on SAD, 31DEC11 AIPS was used.

SAD has several input parameters that affect the number of sources detected. Here, we test the effect of the detection $\mathrm{S} / \mathrm{N}(C P A R M)$, rms threshold, flux residual threshold (DPARM and GAIN), and the size of rmsbox (IMSIZE). The parameter DPARM(3) (along with GAIN added in quadrature) specifies the upper limit for the rms in the fitting box, while $\operatorname{DPARM(7)}$ and GAIN specify the upper limit on the residual flux in the fitting box. GAIN thus defines the fraction of the source flux that is acceptable in the residual image. By default, SAD uses the entire image to find the rms. We used the AIPS task RMSD to prepare rms images using mesh sizes (specified by the IMSIZE parameter) of 5, 10, 20, and 50 beamwidths. Although a decremental search in $\mathrm{S} / \mathrm{N}$ (via $C P A R M$ ) is recommended in the SAD help file, we found that such a search results in multiple sources being fit to a single genuine source during each iteration, especially when the source is extended. Hence, we rejected this recommendation. As in the case of SExtractor, we searched for sources down to $N \sigma$. Note that in SAD, the errors in the flux density are determined theoretically from the image rms (actnoise keyword in the image header).

\footnotetext{
$\overline{14 \text { www.aips.nrao.edu/cgi-bin/ZXHLP2.PL?SAD }}$
}

In Table 7, we list the completeness and reliability of the SAD catalog for different values of the input parameters. We held the input parameters DPARM(3) and DPARM(7) fixed at 1000 and 1 (in units of Jy beam ${ }^{-1}$ ), respectively, in order to get optimum completeness (without significant loss of reliability, as we found later, but possibly at the expense of the correct $\mathrm{S} / \mathrm{N}$ of the detected sources). Due to these large input values, we did not find any change in the results with the GAIN parameter. However, we found that reliability can be traded for completeness by setting smaller values for DPARM(7) and GAIN. In general, the completeness and reliability of SAD increase with the detection threshold. Completeness increases with mesh size, as does reliability, though this quantity decreases significantly as the mesh size is increased from 20 to 50 beamwidths, similar to sfind. Our inspection of the rms image for a mesh size of 50 beamwidths reveals that there are a few pockets where the rms is rather low $(\sim 1 \mu \mathrm{Jy})$. A profusion of false sources (or very low-S/N sources reported to have an $\mathrm{S} / \mathrm{N}$ above the threshold) detected in these pockets is responsible for the markedly reduced reliability for the case of a mesh-size equal to 50 beamwidths. A search with actnoise usually performs at least a few percent worse in terms of completeness and reliability than using a mesh size of 20 beamwidths. Curiously, the completeness and reliability of SAD is $>90 \%$ for a wide range of input parameters tested. We determine the optimum values of input parameters to be $10-20$ beamwidths mesh size and $6 \sigma-7 \sigma$ detection threshold. Searching down to $3 \sigma$ followed by selection of sources greater than $6 \sigma-7 \sigma$ may improve completeness to some extent. Huynh et al. (2011) and Hancock et al. (2012) have not tested SAD on simulated images.

The completeness of SAD with respect to detection $\mathrm{S} / \mathrm{N}$ and for different detection thresholds is shown in the upper-right panel of Figure 8, which depicts the high level of completeness close to the threshold and rapid increase with $\mathrm{S} / \mathrm{N}$ compared to the other algorithms. The upper panel of Figure 9 plots the SAD completeness for optimum input parameters. Due to missed sources throughout the $\mathrm{S} / \mathrm{N}$ spanned, the completeness is seen to hover close to unity, but not quite reaching $100 \%$. The sourcerejection criteria based on $2 \mathrm{D}$ Gaussian fitting in SAD, defined by the several elements of the DPARM input array, is the likely cause for such missed sources. The lower panel of Figure 9 shows the reliability for the optimum values input parameters. As with the case of completeness, the reliability seems to hover close to unity. SAD provides the best reliability for sources $\mathrm{S} / \mathrm{N} \lesssim 20$, beyond which sfind gives better results. Departure of the reliability from unity for a wide range of $\mathrm{S} / \mathrm{N}$ values is due to the fitting of different components to extended sources. Some examples of how SAD fits different kinds of sources in the DR2 image, compared with other algorithms, is shown in Figure 10. Figure 11 shows the $\mathrm{S} / \mathrm{N}$ of sources detected by SAD compared with that of their counterparts in the DR2 catalog. Usually the S/Ns agree with each other, but any disagreement between the two is due to the reported uncertainty in the peak. The peak flux densities reported by SAD match those in the DR2 catalog quite well.

There is a possibility that the superior completeness and reliability of SAD may simply be because the DR2 catalog, against which we are doing all of our comparisons, was constructed from SAD. However, the construction of the DR2 catalog involved much more than running SAD (see Section 3 for more details). In any case, an independent check using simulated sources is warranted. In Figure 12, we compare the completeness and reliability of SAD with other source-finding algorithms on 
the Hancock et al. (2012) simulated image. We find a similar superior behavior of SAD.

\subsubsection{IMSAD (MIRIAD)}

IMSAD $^{15}$ computes the image rms noise by fitting a Gaussian to the image histogram, then searches for contiguous pixels (islands) that are above some cutoff and fits the islands with Gaussian components. For fitting, the routine from the MIRIAD task imfit is used, whereas the island detection is based on the AIPS task SAD. IMSAD can also be used in a mode where the threshold background noise is user-specified. The run time for this source-finding algorithm searching for sources down to $5 \sigma$ in the DR2 image is about $2 \mathrm{~s}$. We used IMSAD version 1998 May 8 from MIRIAD 4.2.3 (optimized for CARMA) to carry out these tests.

As before, we searched for sources down to $N \sigma$ (specified via the clip parameter) and then selected $\geqslant 5 \sigma$ sources. The completeness and reliability were tested using the histogram mode (noplt, hist options set) as well as the user-specified mode (only the noplt option set; rms of $8 \mu \mathrm{Jy}$ used) for backgroundrms determination.

The results of these tests on IMSAD are given in Table 8. The noplt, hist mode usually performs better than the noplt mode at least by a few percent in terms of completeness, whereas the latter mode gives better completeness, again by a few percent. For achieving completeness and a reliability of $80 \%$ or more, the detection threshold needs to be $\geqslant 6 \sigma$. Hence, the optimum use of IMSAD would be in this $\mathrm{S} / \mathrm{N}$ cutoff regime, and the mode in which it is used will depend upon whether completeness or reliability is preferred. Searching down to $3 \sigma$ followed by a rejection of sources below the desired threshold might give better completeness. The reason for the low completeness at a detection threshold of $5 \sigma$ is that several DR2 sources (which are detected by sfind, SAD, and Aegean) close to this threshold are not detected by IMSAD, not even at a reduced $\mathrm{S} / \mathrm{N}$. The reliability at this threshold is also rather low. This can be attributed to several false point sources detected near the edges of the DR2 image, which are mainly low-S/N features mistaken for high$\mathrm{S} / \mathrm{N}$ due to incorrect assumption of the local rms. Since IMSAD does not give the rms for each detected source, one needs to assume this quantity (a constant equal to $8 \mu \mathrm{Jy}$ in our case), and thus the $\mathrm{S} / \mathrm{N}$ calculated in regions of increased rms, e.g., regions close to the edge of the DR2 image, is expected to be erroneous. By using an rms image to find the local rms, this falsedetection problem can be tackled. Another issue related to false sources is that IMSAD does not reject a single sidelobe. All the sidelobes are reported as (genuine) sources. Thus, with respect to false sources detected in noisy regions, missed sources, and sidelobe recognition, IMSAD behaves like SExtractor. The sources detected by IMSAD in some of these scenarios are shown in the image cutouts in Figure 10. Hancock et al. (2012) tested IMSAD on a simulated image and found that its completeness and reliability is lower than other source-finding algorithms. However, through our tests on the DR2 image, it appears that IMSAD has a competitive performance if used with detection thresholds higher than $5 \sigma$.

The completeness of the IMSAD catalog as a function of the detection $\mathrm{S} / \mathrm{N}$ is shown in the lower-left panel of Figure 8. The upper panel of Figure 9 shows the IMSAD completeness for optimum input parameters. Several sources having $10<$ $\mathrm{S} / \mathrm{N}<20$ as well as $\mathrm{S} / \mathrm{N} \gtrsim 100$, which are detected by other

\footnotetext{
15 www.atnf.csiro.au/computing/software/miriad/doc/imsad.html
}

source-finding algorithms, are not detected by IMSAD. This is due to the inability of this algorithm to individually fit blended sources and components of extended sources; IMSAD tends to fit one elongated source for all components (see upper panel of Figure 10). In Figure 9, the reliability of this algorithm is plotted for the optimum input parameters. IMSAD shows reduced reliability between $\mathrm{S} / \mathrm{Ns}$ of 10 and 20, beyond which the reliability roughly flattens off at the $95 \%$ level. The reason for this reduced reliability is twofold. First, several point sources detected close to the edge of the DR2 image have integrated flux densities less than their peak by a factor of a few, clearly indicating unphysical fitting of sources. These false sources can easily be rejected by using a peak-to-integrated flux density ratio criterion. Second, IMSAD does not break up islands into components as mentioned above, resulting in extended source positions that are substantially different from those listed in the DR2 catalog. This was also found in the tests carried out by Hancock et al. (2012), which resulted in several false detections. Figure 11 shows the $\mathrm{S} / \mathrm{N}$ of IMSAD sources compared with their counterparts in the DR2 catalog. The assumed constant rms as well as the somewhat lower peak flux density reported by IMSAD (for extended sources) are responsible for the lower $\mathrm{S} / \mathrm{N}$ with respect to the DR2 sources.

\subsubsection{Aegean}

Aegean ${ }^{16}$ uses the FloodFill algorithm, which separates the foreground pixels from the background and groups them into islands. These "islands" are then passed on to the source characterization stage. Each island of pixels is fit with multiple Gaussian components. The number of components to be fit is determined from a surface-curvature map (cmap), derived from the input image with a Laplacian transform. Aegean thus performs a well-constrained multiple Gaussian fitting. A detailed description of Aegean and its implementation can be found in Hancock et al. (2012), where the authors demonstrate this source-finding algorithm on a simulated image to produce catalogs for better reliability and completeness than other source-finding algorithms. The run time for Aegean searching for sources down to $5 \sigma$ in the DR2 image is about 4 minutes (using two CPU cores). We used Aegean r706 (2012 July 25 release) for testing this source-finding algorithm.

We tested the effect of the parameters: (i) innerclip (seedclip) and outerclip (floodclip); and (ii) csigma on the completeness and reliability of the Aegean catalog. Innerclip defines the sigma clipping (lower limit) which is used for the detection ("seeding") of islands, whereas outerclip defines that used for reporting ("flooding") the islands in the catalog. csigma is the sigma clipping parameter for the curvature map. Note that the r706 version of Aegean uses an immutable mesh-size of 20 beamwidths. We used innerclip $=$ outerclip for our tests. Setting the innerclip to $1 \sigma$ lower than the outerclip gives results that are alike, equating these two clipping parameters, while using a $1 \sigma$ lower outerclip than innerclip seems to give a few percent better completeness and a slightly reduced reliability.

Our results for Aegean are given in Table 9. csigma does not have a significant effect on either the completeness or reliability. As with our tests on other source-finding algorithms, we searched for sources down to $N \sigma$. The best completeness and reliability require a $7 \sigma$ or higher detection threshold. Using a $3 \sigma$ detection threshold followed by a selection of greater than $7 \sigma$ might give better completeness at the cost

\footnotetext{
$\overline{16}$ www.physics.usyd.edu.au/\% 7Ehancock/index.php/Programs/Aegean
} 
of reliability. Surprisingly, the completeness and reliability are not $>95 \%$, as seen in the tests with the Hancock et al. (2012) simulated image. This is because Aegean performs well with isolated point sources, but not quite with extended or blended ones. For extended sources, Aegean tends to fit some of the components at unexpected locations and with quite elongated Gaussians (the fitting-error flag is also set for these components). Numerous small (but unphysical; size equal to the synthesized beam) components are also reported for several extended sources. Since Hancock et al. (2012) have demonstrated that Aegean successfully fits all the components of blended simulated sources, we think that diffuse components and imaging artifacts such as negative sidelobes adversely affect the curvature map produced by this source-finding algorithm, which in turn determines the sources reported. An example of such a case is given in the upper panel of Figure 10. Note that in the latest release of Aegean (r808; 2013 February 13), this situation is somewhat improved, since some of the elongated components from $\mathrm{r} 706$ are reported with more realistic source sizes (and also without any fitting-error flags) and relatively fewer small-size components are fit.

Figure 8 shows the completeness of the Aegean catalog as a function of $\mathrm{S} / \mathrm{N}$ of sources in the DR2 catalog, and the upper panel of Figure 9 shows the completeness for csigma $=1 \sigma_{\mathrm{cmap}}$, and searching for sources down to $3 \sigma$. Aegean completeness appears to be quite good except for sources below $7 \sigma$ in the DR2 catalog, which are detected at a decreased S/N (below 5) and hence are rejected from the catalog, and for a few components of extended sources above $\sim 100 \sigma$, which are reported to have positions not matching those in the DR2 catalog. The decreased $\mathrm{S} / \mathrm{N}$ reported for $5 \sigma-7 \sigma$ sources is due to a slightly decreased peak flux density and a slightly increased rms with respect to the DR2 catalog. Figure 9 plots the reliability using csigma $=1 \sigma_{\mathrm{cmap}}$ and searching for sources down to $3 \sigma$. The significant deviation of reliability from unity for $\mathrm{S} / \mathrm{N}>10$ sources results from the several (false) small and elongated components reported for extended sources, as mentioned above. Figure 11 shows the $\mathrm{S} / \mathrm{N}$ of sources detected by Aegean compared with the corresponding sources in the DR2 catalog. The disagreement in $\mathrm{S} / \mathrm{N}$ for the $5 \sigma-7 \sigma$ sources in the DR2 catalog has been explained above. For $\mathrm{S} / \mathrm{N}>200$ sources, the peak flux densities agree quite well between the Aegean and DR2 catalogs, but the rms reported by Aegean is consistently higher, which causes the disagreement at the high-S/N end of the diagram.

\subsubsection{Summary of Results from the Efficiency Tests}

We find remarkable differences between algorithms in terms of components fitted for extended sources, sidelobe rejection, and point sources detected in regions where the rms is appreciably larger than the mean rms. Our results for completeness and reliability are broadly similar to those of Huynh et al. (2011) and Hancock et al. (2012). For applications that need both completeness and reliability, sfind and Aegean, are good. Additionally, we found that the SAD algorithm within the widely available AIPS package had a better performance. IMSAD also gives good completeness and reliability for detection thresholds $\geqslant 6 \sigma$. For transient searches, reliability takes preference over completeness, since false positives are likely to consume follow-up resources. Most transient projects are likely to be searching in near real time. However, in this particular project, we were fortunate to have a deep reference image that was more than three times deeper than the single-epoch images. This allowed us to study reliability with real (rather than synthetic) data sets.
From Figure 9, we see that the reliability of sfind is better than that of SExtractor, IMSAD, and Aegean except for S/N near 8. However, the best reliability is provided by SAD.

\subsection{Transient Candidate Search}

Using the best-performing source-finding algorithms from Section 4.1.6 and their optimum parameter values, we carried out a search for transient radio sources over all epochs. We ran sfind, SAD, IMSAD, and Aegean on single-epoch images and obtained 49 single-epoch catalogs for each algorithm. We required that any potential transient candidate identified in the single-epoch catalog obey the following constraints.

1. The source is not found in the reference catalog (within $2^{\prime \prime}$ ) of persistent sources. The reference catalog was constructed similarly to DR2 but the sources were selected up to the $20 \%$ power point of the beam (i.e., $\theta<21$ '.5 radius) of each pointing rather than the $34^{\prime}$ interior region shown in Figure 1.

2. The source is at least a $7 \sigma$ detection.

3. It is a genuine point-like source, i.e., it has

(a) $0.9<S_{\text {int }} / S_{\text {peak }}<1.5$

(b) $a<2^{\prime \prime} .8 \times 1.5, b<1^{\prime \prime} .6 \times 1.5$ ( $a=$ major axis, $b=$ minor axis; recall that the synthesized beam is $\left.2^{\prime \prime} .8 \times 1^{\prime \prime} .6\right)$.

4. The source is at least 20 synthesized beams $(20 \times$ geometric mean of FWHMs; 42") away from the nearest

(a) bright source $(>500 \mu \mathrm{Jy})$, so that any sidelobe emission is rejected

(b) extended source.

The multiplicative factor of three-halves used in the selection of point sources, as well as the distance of 20 beamwidths used to constrain the proximity from bright and extended sources, is somewhat arbitrary, but is based on several iterations of our transient search code and inspection of the cutouts of the resulting transient candidates. By investigating how the major axes of sources increase with their distance from the pointing center, we found that $\theta \simeq 21^{\prime} .5$ appears also to be the threshold beyond which bandwidth smearing coupled with our constraints on the major (and minor) axes start rejecting genuine point sources.

Due to the large number of synthesized beams searched $\left(n=1.8 \times 10^{7}\right)$ in this data set, there is a modest probability that a transient candidate is due to noise. ${ }^{17}$ We thus carried out an analysis similar to Frail et al. (2012, see Appendix A of that paper) to determine the $\mathrm{S} / \mathrm{N}$ above which the probability of having the highest value of $n$ Gaussian random numbers is $\leqslant 1 \%$. This corresponds to an $\mathrm{S} / \mathrm{N}$ of 6.1 . However, following the recommendation of Frail et al. (2012) to have a higher S/N cutoff when the noise was not strictly Gaussian, we chose $7 \sigma$ as the lower limit for finding transients.

The search method outlined above may miss transients that are bright enough to be present in the reference catalog. Therefore, we also searched (with similar constraints as above) for sources that are detected in the reference catalog of persistent sources and detected in only one of the single-epoch catalogs.

For sf ind, we adopted the parameters $\alpha=10$ and $r m s b o x=$ 20 for our transient search. We found five candidates that are present only in a single epoch above the $7 \sigma$ detection threshold. They also show up in the reference catalog. However, all of these 17 From theory, we know that the statistics of beam values of interferometric
maps should follow a Gaussian distribution. 
candidates seem to be variable but persistent, and are detected at a low $\mathrm{S} / \mathrm{N}(\sim 3 \sigma-5 \sigma)$ in other epochs. Thus, we do not find any transient with sfind.

For Aegean, we used a clipping level of $7 \sigma$ and a curvaturemap cutoff of $1 \sigma$. We discarded all the transients corresponding to islands which were too small to give a six-parameter Gaussian fit (sources with flag 10000 or 00100 set) since otherwise we were dealing with a large number of transients. Using these constraints, we found one candidate which is present in the reference catalog and is a persistent source detected at a largely reduced $\mathrm{S} / \mathrm{N}$ in other epochs. Accordingly, Aegean does not yield any transients.

For $\mathrm{SAD}$, we searched for sources down to $3 \sigma$ with an rmsbox of 20 beamwidths, and used the input parameters $\operatorname{DPARM}(3), \operatorname{DPARM}(7)=(1000,1)$ to ensure optimum completeness and reliability based on our efficiency test. We do not find any transients with SAD.

With IMSAD, we searched for sources down to $7 \sigma$ with the histogram option set. The several sources that are found to be transient candidates are only variables at our detection limit, as is evident through visual inspection of the images. Thus, there are no transients reported by IMSAD.

\section{DISCUSSION AND CONCLUSIONS}

In this paper, we have explored the time-domain properties of a $1.4 \mathrm{GHz}$ survey made toward the E-CDFS region. Six mosaic pointings toward E-CDFS were taken in 49 separate epochs over a period of three months. Single-epoch images allow us to explore the transient and variable radio sky at sub-mJy levels on timescales of days, weeks, and months. We will now use these data to assess the degree of variability (Section 5.1) and the transient rate (Section 5.2) of the radio sky, and predict what will be seen by future wide-field surveys (Section 5.3).

\subsection{Comparison of Variability with Previous Surveys}

We found in our study that only a small fraction $(7 / 599=$ $\left.1.2_{-0.7}^{+1.2} \%\right)$ of the point sources in the E-CDFS showed any significant variability on day-week-month timescales. Evidently, the sub-mJy radio sky at $1.4 \mathrm{GHz}$ is not highly variable. The only previous sub-mJy study at $1.4 \mathrm{GHz}$ was from a single deep pointing toward the Lockman Hole. With sampling timescales of 19 days, Carilli et al. (2003) found less than $2 \%$ of sources above $0.1 \mathrm{mJy}$ to be highly variable.

Our findings at sub-mJy levels are consistent with several previous $1.4 \mathrm{GHz}$ studies at higher flux density thresholds. Thyagarajan et al. (2011) analyzed the $8444 \mathrm{deg}^{2}$ of the Faint Images of the Radio Sky at Twenty cm (FIRST) survey and found that only $0.5 \%(1627 / 279407)$ of sources above $1 \mathrm{mJy}$ varied significantly on timescales of minutes to years. Frail et al. (1994) imaged a $2^{\circ}$ region toward a gamma-ray burst on timescales of 1-96 days and found that fewer than $\sim 1 \%$ of the sources above a flux density of $3.5 \mathrm{mJy}$ were strongly variable. de Vries et al. (2004) imaged a $120.2 \mathrm{deg}^{2}$ area of Sloan Digital Sky Survey Stripe 82 finding $\leqslant 1.4 \%$ (123/9086) of the radio sources to be strongly variable (i.e., $>4 \sigma$ ) above flux densities of $2 \mathrm{mJy}$ on a 7 year timescale. Ofek \& Frail (2011) do a two-epoch comparison of FIRST and NVSS point sources brighter than $5 \mathrm{mJy}$ and find that only $0.1 \%$ (43/4367) vary by more than $4 \sigma$ over timescales ranging from about 300 to 1700 days. Bannister et al. (2011) analyzed 22 years and $2775 \mathrm{deg}^{2}$ of MOST observations at $0.84 \mathrm{GHz}$ and found only
$0.17 \%(53 / 29730)$ strong variables above $14 \mathrm{mJy}$ on timescales of days to minutes to years. Finally, Croft et al. (2011) used the Allen Telescope Array to survey a $690 \mathrm{deg}^{2}$ area at $1.4 \mathrm{GHz}$. They compared their catalog to the NVSS, finding that $0.1 \%$ $(6 / 4408)$ of the sources were highly variable on a timescale of 15 years.

In Figure 13, we plot the differential source counts for the persistent radio sky at $1.4 \mathrm{GHz}$, normalized in the usual way by the Euclidean rate (Huynh et al. 2005). The steep evolution of the AGN with decreasing flux density is apparent, as is the flattening of the source counts near $1 \mathrm{mJy}$. The fractional variability appears to be low, at a level of one percent, among the sources greater than $100 \mu \mathrm{Jy}$.

Comparing the variability properties at $1.4 \mathrm{GHz}$ of our sub-mJy population with those at higher flux densities, we find no obvious change in the fraction of strong variables. This is despite the fact that radio-loud AGNs (which dominate at mJy levels) diminish in importance as radio-quiet AGNs and star-forming galaxies begin to populate the radio sky at lower flux densities, the latter constituting about $50 \%$ of the sub-mJy radio sky (Smolčić et al. 2008; Padovani 2011). On the other hand, in a sample of brightness temperature-limited radio sources, it would be expected that the variability would increase with decreasing flux density.

Our optical identifications for the seven radio variables show a mix of AGNs and star-forming galaxies. Six of these are consistent with a nuclear source based on the carefully matched astrometry. There is no evidence from this sub-mJy sample that we are seeing a new source of variable radio emission, such as supernovae, gamma-ray bursts, etc. that would be expected to be offset from the nucleus.

Radio variability appears to be a strong function of frequency. The best study to compare to this one is the $5 \mathrm{GHz}$ survey of Ofek et al. (2011), since it used a similar observing cadence and identical statistical measures of variability. In this case, it was found that $\sim 30 \%$ of point sources brighter than $1.5 \mathrm{mJy}$ at $5 \mathrm{GHz}$ were variable.

Some caution is warranted when comparing fractional variability between different experiments. Differences in the cadence, integration time, duration, and angular resolution will have a tendency to reduce the variability amplitude. For example, the low angular resolution of some surveys may have the effect of reducing the strong variability from compact radio sources embedded in diffuse emission. This current survey with a synthesized beam of $2^{\prime \prime} .8 \times 1^{\prime \prime} .6$ has the highest angular resolution of any previous variability survey (see Ofek et al. 2011).

Another factor to consider is that the degree of variability that can be detected in a given experiment, as measured by the modulation index $m$, is a function of the $\mathrm{S} / \mathrm{N}$. A source in our present survey would have to have a mean flux density of $1 \mathrm{mJy}$ in order to detect a $10 \%$ modulation, while at the $5 \sigma$ limit of the DR2 catalog $(\sim 40 \mu \mathrm{Jy})$ a source would have to have $m \geqslant 1.5$ in order to be identified as a significant variable. No strong variables (i.e., $m>0.5$ ) were identified in our survey, but only the about 90 sources in the DR2 catalog are bright enough $(\geqslant 126 \mu \mathrm{Jy})$ to have been identified as a strong variable. In either case, the fraction of significant or strong variables is less than a few percent of the sample.

The most robust conclusion that can be drawn is that the variable radio sky at $1.4 \mathrm{GHz}$ appears to be relatively quiet, with only a fraction of a percent of sources varying substantially over a wide range of flux densities and timescales. 


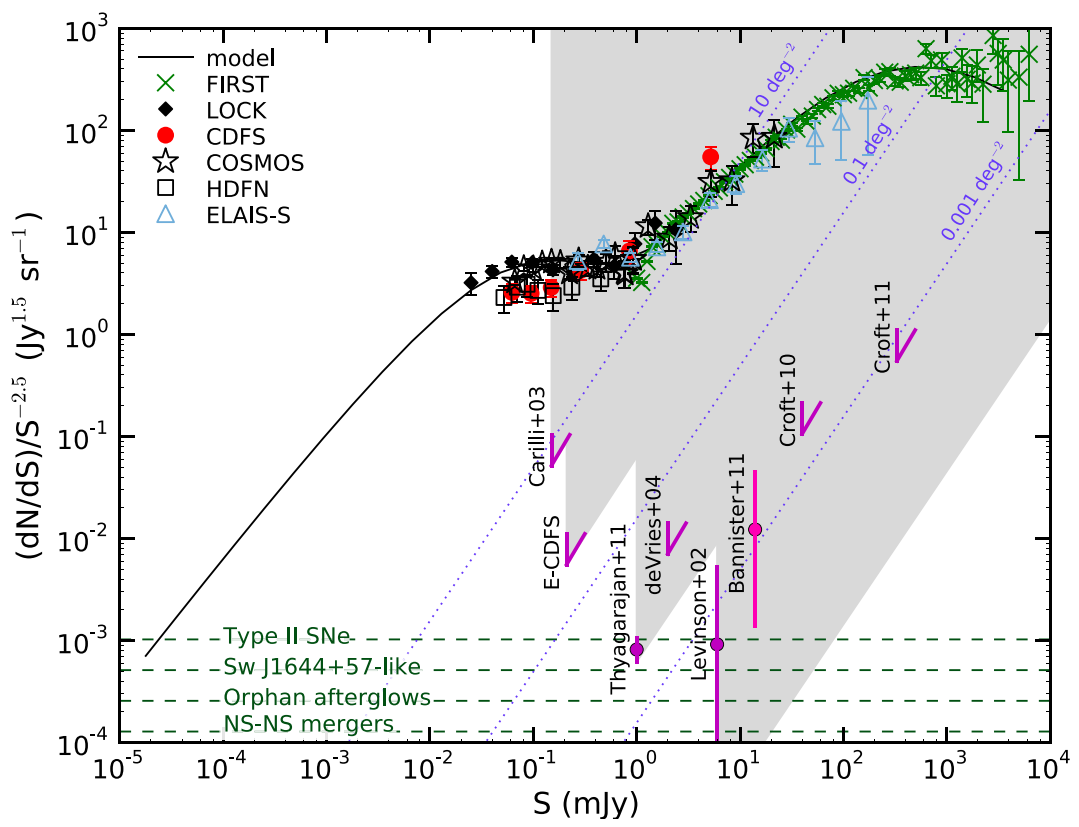

Figure 13. Normalized 1.4 GHz differential radio-source counts for persistent sources from de Zotti et al. (2010) and the normalized areal density of transients (or limits) as a function of the flux density for various surveys at this frequency. The Bannister et al. (2011) survey at $0.84 \mathrm{GHz}$ is colored differently than the other surveys. Most of the surveys are upper limits (wedge symbols) and the sampled phase space is shown by the gray shaded area. Upper limits from Frail et al. (1994) and Bower \& Saul (2011) do not explore any new part of the phase space (non-gray area), and hence have been left out of this diagram. Our upper-limit is labeled as "E-CDFS." Three surveys have transient detections so far, the $2 \sigma$ error bars for which are shown according to Gehrels (1986). Note that Thyagarajan et al. (2011) and Bannister et al. (2011) may have identified a few strong variables as transients (see Section 5.2), which would make their detections move downward on this plot. The black solid line is the model for AGNs and star-forming galaxies from Condon (1984). Lines of constant areal density are shown as blue dotted lines. The horizontal dashed lines are estimates for the areal density for known and expected classes of long-duration radio transients taken directly from Frail et al. (2012). The areal density for Swift J1644+57-like tidal disruption events has been modified according to Berger et al. (2012) to reflect their true rate at $1.4 \mathrm{GHz}$. Upper limits from the ASKAP-VAST surveys are estimated to be an order of magnitude or more below the rate of orphan gamma-ray burst afterglows, and to have an rms sensitivity ranging between $10 \mu \mathrm{Jy}$ and $0.5 \mathrm{mJy}$.

(A color version of this figure is available in the online journal.)

\subsection{Limits on Transient Areal Density and Rate}

We searched our multi-epoch data for transients but found none. The search was conducted on each image out to a radius of 21.5 from the pointing center. The single-epoch area out to that radius is $0.40 \mathrm{deg}^{2}$, or a total area of $20 \mathrm{deg}^{2}$ for all 49 epochs. However, the sensitivity of the VLA antennas is not uniform across this area. The primary beam response is well described by a Gaussian with a half-width to half-maximum of $15^{\prime}$, falling to $20 \%$ response at our search radius of $21^{\prime} .5$. At the pointing center, the $7 \sigma$ flux density limit was approximately $210 \mu \mathrm{Jy}$ for each epoch.

In order to calculate a limit on the areal density of any putative transient population, we follow Ofek et al. (2011) and parameterize the source number-count function as a power law of the form

$$
\kappa(>S)=\kappa_{0}\left(S / S_{0}\right)^{-\gamma},
$$

where $S$ is the peak flux density, $\kappa(>S)$ is the sky surface density of sources brighter than $S, \kappa_{0}$ is the sky surface density of sources brighter than $S_{0}$, and $\gamma$ is the power-law index of the source number-count function. We assume for simplicity a homogeneous source distribution in a Euclidean universe so that $\gamma=3 / 2$. The one-sided $2 \sigma$ upper limit on the areal density is three events (Gehrels 1986). Therefore, using Equation (C5) in Ofek et al. (2011), we find that the $2 \sigma$ upper limit on areal density to a flux limit of $210 \mu \mathrm{Jy}$ is $18.0 \mathrm{deg}^{-2}$ per epoch. Given that we have 49 epochs, the $2 \sigma$ upper limit on the areal density is $\kappa(>0.21 \mathrm{mJy})<0.37 \mathrm{deg}^{-2}$. We can further estimate an upper limit on the transient rate assuming a duration $t_{\text {dur }}$ less than the shortest time between epochs of $\Re(>0.21 \mathrm{mJy})<268\left(t_{\text {dur }} / 0.5 \text { day }\right)^{-1} \mathrm{deg}^{-2} \mathrm{yr}^{-1}$.

Our upper limit on the areal density of transient sources at sub-mJy levels can be compared with the predictions based on previous surveys. The Bower et al. (2007) survey is a useful benchmark since their areal density dominates all known classes of transients. Adopting their measured two epoch rate of $\kappa(>0.37 \mathrm{mJy})=1.5 \mathrm{deg}^{-2}$ and assuming a Euclidean source distribution (i.e., $\gamma=3 / 2)$, we predict $\kappa(>0.21 \mathrm{mJy})=$ $3.5 \mathrm{deg}^{-2}$ at the flux density limit of our current survey.

An alternative way to look at our results is to compare our null detection to the expected number of Bower et al. transients expected in our data set. We use the parameterization of Fender \& Bell (2011) for the predicted Bower et al. transient rate as a function of flux density,

$$
\log \left(\frac{\kappa}{\mathrm{deg}^{-2}}\right)=-1.5 \log \left(\frac{S_{v}}{\mathrm{Jy}}\right)-5.13,
$$

where $\kappa$ is the snapshot rate, and $S_{v}$ denotes the detection threshold of the observations at the pointing center (i.e., $7 \sigma=$ $210 \mu \mathrm{Jy})$. Integrating both sides of Equation (5) over the azimuthal angle and in $\theta$ out to 21'.5, we get about 0.42 transients per epoch if the Bower et al. (2007) transients are real. Since we have 49 epochs, we expect to have about 21 Bower et al. transients in our E-CDFS data set.

Our search on the E-CDFS field suggests that the areal density of radio transients is an order of magnitude or more below the rate measured by Bower et al. (2007; i.e., $<0.37 \mathrm{deg}^{-2}$ versus $3.5 \mathrm{deg}^{-2}$ ). Alternatively, we find a $2 \sigma$ upper limit of $<3$ 
Table 10

Telescope Specifications

\begin{tabular}{lcccrccc}
\hline \hline Telescope & $\begin{array}{c}\text { BW } \\
(\mathrm{MHz})\end{array}$ & $\begin{array}{c}\Omega \\
\left(\mathrm{deg}^{2}\right)\end{array}$ & $\begin{array}{c}D \\
(\mathrm{~m})\end{array}$ & $N$ & $\epsilon_{e}$ & $\begin{array}{c}T_{\text {sys }} \\
(\mathrm{K})\end{array}$ & SS \\
\hline VLA & 512 & 0.25 & 25 & 27 & 0.5 & 26 & 1.0 \\
ASKAP & 300 & 30 & 12 & $18(36)$ & 0.8 & 50 & $1.1(4.6)$ \\
Apertif & 300 & 8 & 25 & 13 & 0.75 & 70 & 1.3 \\
MeerKAT & 750 & 1 & 13.5 & 64 & 0.7 & 30 & 4.1 \\
\hline
\end{tabular}

Notes. Here, BW is bandwidth in $\mathrm{MHz}, \Omega$ is the field of view in $\operatorname{deg}^{2}, D$ is the antenna diameter in meters, $N$ is the number of antennas in the array, $\epsilon_{e}$ is the aperture efficiency, $T_{\text {sys }}$ is the system temperature in Kelvin, and SS is the survey speed normalized to the VLA.

transients, while the predicted number is $\sim 21$ transients. Our work therefore appears to support that of Frail et al. (2012), which found from a reanalysis of the Bower et al. data that the transient rate was as much as an order of magnitude smaller than previously reported. This conclusion would be more robust if the spectral index of the putative transient population was better known. Ofek et al. (2010) was able to use other surveys to constrain the spectral index $\alpha>0$ (where $S_{v} \propto v^{\alpha}$ ). Since the Bower et al. (2007) rates were derived based on observations made mostly at $5 \mathrm{GHz}$, our only data provide strong constraints for $0 \leqslant \alpha \leqslant 1.1$. A population of optically thick $\alpha \simeq 5 / 2$ sources with a rate similar to that of Bower et al. (2007) would be undetected in our 1.4 GHz E-CDFS fields.

Many of the same variability surveys discussed in Section 5.1 were also sensitive to transients. Superposed on the radiosource count plot of Figure 13 are the results of several of these transients surveys. Light gray shaded areas represent the transient phase space covered by each of the surveys and white space represents open phase space for future narrow-deep or wide-shallow surveys. With few exceptions, most of these transient surveys result in upper limits. Also shown are the normalized areal density of several known and expected classes of long-duration radio transients, based on Frail et al. (2012). The nominal rates for the putative Bower et al. (2007) sources are about two orders of magnitude above the tidal disruption for Sw J1644+57-like objects. Thyagarajan et al. (2011) define a transient as having either a single detection in the analyzed epochs or the highest flux density five times greater than the next highest one (detection/upper-limit). Thus, Thyagarajan et al. (2011) and two-epoch surveys like Bannister et al. (2011) may identify a strong variable as a true transient, which will move the source count of detected transients lower in Figure 13.

\subsection{Future Radio Surveys}

There are several facilities built or under construction that will be capable of synoptic imaging at $1.4 \mathrm{GHz}$. All of these facilities have the exploration of the time domain as part of their core science programs. The Australian Square Kilometer Array Pathfinder (ASKAP) and the Apertif instrument on the Westerbork Synthesis Radio Telescope (WSRT) will be using focal plane array technology to image an instantaneous FoV of $30 \mathrm{deg}^{2}$ and $8 \mathrm{deg}^{2}$, respectively (Murphy et al. 2012; Oosterloo et al. 2010). South Africa is building MeerKAT, an array of $6413.5 \mathrm{~m}$ diameter dishes, with an FoV of $1 \mathrm{deg}^{2}$ at $1.4 \mathrm{GHz}$ (Booth et al. 2009). Finally, there is the newly refurbished Karl G. Jansky Array (VLA), which has $2725 \mathrm{~m}$ dishes with an FoV of $0.25 \mathrm{deg}^{2}$ at $1.4 \mathrm{GHz}$ (Perley et al. 2011).

We list the capabilities of each of these telescopes in Table 10. Survey speed (SS), normalized here to the VLA, is a useful figure of merit for inter-comparison of survey capabilities of long duration transients and is expressed as

$$
\mathrm{SS} \propto \mathrm{BW} \times \Omega\left(A_{e} / T_{\mathrm{sys}}\right)^{2},
$$

where $\mathrm{BW}$ is the bandwidth, $\Omega$ is the $\mathrm{FoV}, A_{e}$ is the total collecting areas times the aperture efficiency $\epsilon_{e}$, and $T_{\text {sys }}$ is the antenna system temperature (Cordes 2008). The relative SSs are only approximate since some of the system parameters for MeerKAT and ASKAP have not been confirmed with on-thesky testing. Likewise, we have assumed that radio-frequency interference (RFI) limits the VLA bandwidth to only $50 \%$ of its maximum BW. Larger fractions are achievable in the more extended array configurations and with better RFI excision of the data. We have calculated the ASKAP SS with both the 18 phased array feeds which are currently funded and with the full 36 as originally specified. Despite these uncertainties, it is clear from Table 10 that to within factors of a few, these are all powerful wide-field imaging facilities.

To illustrate these survey capabilities and compare them to what we currently know about the transient and variable radio sky, we will use the example of an electromagnetic (EM) counterpart search for gravitational waves (GWs). For a good overview of the topic of EM-GW searches and the main issues, we refer the reader to Metzger \& Berger (2012) and Nissanke et al. (2013).

Long-duration radio emission has been predicted to originate from several sources caused by the merger of a neutron star binary including the merger shock (Kyutoku et al. 2012), afterglow emission from the beamed outflow in the relativistic and non-relativistic phases (Metzger \& Berger 2012), and from quasi-isotropic, mildly relativistic outflows ejected during the merger (Nakar \& Piran 2011; Piran et al. 2012). All of these mechanisms depend on the amount of energy put into shocked material and the density of the ambient medium. Predicted flux densities and timescales therefore vary over a wide range. We take as an example the detection of a signal with a peak flux density of $100 \mu \mathrm{Jy}$. Such a signal might be expected to occur for an ambient medium with a density of $0.1 \mathrm{~cm}^{-3}$ on day-long timescales for a merger shock, or on year-long timescales for mildly relativistic ejecta. We note that if short-duration gammaray bursts are the dominant population of neutron star mergers, then the predicted radio signal would be difficult to detect with the telescopes in Table 10, given the canonical energy and ambient density inferred for this population (Fong et al. 2012; Metzger \& Berger 2012).

The median sky localization of a gravitational-wave source will be $60 \mathrm{deg}^{2}$ with a three-element GW network, and $7 \mathrm{deg}^{2}$ with a five-element GW network (Nissanke et al. 2013). With such large error boxes, the main challenge for the identification of an EM-GW counterpart will be to distinguish it from the foreground of false positives. Optical-only searches for EM-GW counterparts are expected to be overwhelmed by false positives at the required depth of 22-23 mag and special strategies are required (Nissanke et al. 2013). However, as we have shown from this paper, the radio sky at $1.4 \mathrm{GHz}$ is relatively quiet. Integrating the differential source counts in Figure 13 using the fit from Huynh et al. (2005), we estimate that the number of persistent radio sources above $100 \mu \mathrm{Jy}$ to be $910 \mathrm{deg}^{-2}$. We have estimated that the fraction of strong variables, on a wide range of timescales, is likely to be one percent or less, or nine strong variables per square degree. Further, the radio variables that we have seen to date have all been nuclear sources (Section 3.3). Such variable or transient sources could be easily 
rejected as EM-GW counterparts since significant offsets are predicted from the host galaxy based on binary neutron-star population synthesis models and measurements from short-hard gamma-ray bursts (Belczynski et al. 2006; Fong et al. 2010). Lastly, the number of unrelated transients is also expected to be much less. Our derived limit on the transient areal rate of $\kappa(>0.21 \mathrm{mJy})<0.37 \mathrm{deg}^{-2}$ translates to $\mathrm{a}<1.1 \mathrm{deg}^{-2}$ at $100 \mu \mathrm{Jy}$ (Section 5.2) for a Euclidean distribution. The limit on the known transient populations is even smaller (Frail et al. 2012).

We believe that the multi-wavelength approach which we have taken here should inform future searches. For the radio variables that we found in the E-CDFS, we were able to identify the source of the emission using optical images and spectra (Section 3.3). A similar strategy could be employed to identify false positives for the small number of radio variables or transient sources identified in EM-GW counterpart searches. Whether or not this approach will ultimately lead to a robust EM-GW counterpart detection is uncertain, but in terms of characterizing the variables, minimizing false positives, and getting an early sense on the nature of transients, we suggest that joint radio-optical searches will be fruitful for exploring the dynamic sky.

M. Kunal wishes to thank Rick Perley, Eric Greisen, Sanjay Bhatnagar, Andrea Petric, Margherita Bonzini, Bill Cotton, and Paul Hancock for useful discussions. The National Radio Astronomy Observatory is a facility of the National Science Foundation operated under cooperative agreement by Associated Universities, Inc. S.R.K.'s research in part is supported by NASA and NSF. This research has made use of NASA's Astrophysics Data System, Vizier, and NED. We thank the anonymous referee for useful comments.

\section{REFERENCES}

Afonso, J., Mobasher, B., Koekemoer, A., Norris, R. P., \& Cram, L. 2006, AJ, 131,1216

Assef, R. J., Kochanek, C. S., Brodwin, M., et al. 2010, ApJ, 713, 970

Bannister, K. W., Murphy, T., Gaensler, B. M., Hunstead, R. W., \& Chatterjee, S. 2011, MNRAS, 412, 634 (erratum 418, 2813)

Belczynski, K., Perna, R., Bulik, T., et al. 2006, ApJ, 648, 1110

Bell, M. E., Fender, R. P., Swinbank, J., et al. 2011, MNRAS, 415, 2

Berger, E., Price, P. A., Cenko, S. B., et al. 2005, Natur, 438, 988

Berger, E., Zauderer, A., Pooley, G. G., et al. 2012, ApJ, 748, 36

Bonzini, M., Mainieri, V., Padovani, P., et al. 2012, ApJS, 203, 15

Booth, R. S., de Blok, W. J. G., Jonas, J. L., \& Fanaroff, B. 2009, arXiv:0910.2935

Bower, G. C., \& Saul, D. 2011, ApJL, 728, L14

Bower, G. C., Saul, D., Bloom, J. S., et al. 2007, ApJ, 666, 346

Bridle, A. H., \& Schwab, F. R. 1989, in ASP Conf. Ser. 6, Synthesis Imaging in Radio Astronomy, ed. R. A. Perley, F. R. Schwab, \& A. H. Bridle (San Francisco, CA: ASP), 247

Cameron, P. B., Chandra, P., Ray, A., et al. 2005, Natur, 434, 1112

Camilo, F., Ransom, S. M., Halpern, J. P., et al. 2006, Natur, 442, 892

Carilli, C. L., Ivison, R. J., \& Frail, D. A. 2003, ApJ, 590, 192

Cenko, S. B., Krimm, H. A., Horesh, A., et al. 2012, ApJ, 753, 77

Chary, R., \& Elbaz, D. 2001, ApJ, 556, 552

Condon, J. J. 1984, ApJ, 284, 44

Condon, J. J. 2007, in ASP Conf. Ser. 380, At the Edge of the Universe: Latest Results from the Deepest Astronomical Surveys, ed. J. Afonso, H. C. Ferguson, B. Mobasher, \& R. Norris (San Francisco, CA: ASP), 189

Condon, J. J., Cotton, W. D., \& Broderick, J. J. 2002, AJ, 124, 675

Condon, J. J., Cotton, W. D., Fomalont, E. B., et al. 2012, ApJ, 758, 23

Cordes, J. M. 2008, in ASP Conf. Ser. 395, Frontiers of Astrophysics: A Celebration of NRAO's 50th Anniversary, ed. A. H. Bridle, J. J. Condon, \& G. C. Hunt (San Francisco, CA: ASP), 225
Cotton, W. D., \& Perley, R. 2010, OBIT Development Memo Series No. 17, ftp://ftp.cv.nrao.edu/NRAO-staff/bcotton/Obit/EVLABeam.pdf

Cotton, W. D., \& Peters, W. 2011, OBIT Development Memo Series No. 25, ftp://ftp.cv.nrao.edu/NRAO-staff/bcotton/Obit/FDR.pdf

Croft, S., Bower, G. C., Keating, G., et al. 2011, ApJ, 731, 34

Damen, M., Labbé, I., van Dokkum, P. G., et al. 2011, ApJ, 727, 1

Desai, V., Armus, L., Spoon, H. W. W., et al. 2007, ApJ, 669, 810

de Vries, W. H., Becker, R. H., White, R. L., \& Helfand, D. J. 2004, AJ, 127,2565

de Zotti, G., Massardi, M., Negrello, M., \& Wall, J. 2010, A\&ARv, 18, 1

Donley, J. L., Koekemoer, A. M., Brusa, M., et al. 2012, ApJ, 748, 142

Fender, R. P., \& Bell, M. E. 2011, BASI, 39, 315

Fong, W., Berger, E., \& Fox, D. B. 2010, ApJ, 708, 9

Fong, W., Berger, E., Margutti, R., et al. 2012, ApJ, 756, 189

Fox, D. B., Frail, D. A., Price, P. A., et al. 2005, Natur, 437, 845

Frail, D. A., Kulkarni, S. R., Hurley, K. C., et al. 1994, ApJ, 437, 43

Frail, D. A., Kulkarni, S. R., Ofek, E. O., Bower, G. C., \& Nakar, E. 2012, ApJ, 747,70

Gaensler, B. M., \& Hunstead, R. W. 2000, PASA, 17, 72

Gaensler, B. M., Kouveliotou, C., Gelfand, J. D., et al. 2005, Natur, 434, 1104

Gehrels, N. 1986, ApJ, 303, 336

Giavalisco, M., Ferguson, H. C., Koekemoer, A. M., et al. 2004, ApJ, 600, 93

Hancock, P. J., Murphy, T., Gaensler, B. M., Hopkins, A., \& Curran, J. R. 2012, MNRAS, 422, 1812

Hopkins, A. M., Miller, C. J., Connolly, A. J., et al. 2002, AJ, 123, 1086

Huynh, M., Hopkins, A., Norris, R., et al. 2012, PASA, 29, 229

Huynh, M. T., Hopkins, A. M., Lenc, E., et al. 2012, MNRAS, 426, 2342

Huynh, M. T., Jackson, C. A., Norris, R. P., \& Prandoni, I. 2005, AJ, 130, 1373

Hyman, S. D., Lazio, T. J. W., Kassim, N. E., et al. 2005, Natur, 434, 50

Hyman, S. D., Wijnands, R., Lazio, T. J. W., et al. 2009, ApJ, 696, 280

Johnston, S., Taylor, R., Bailes, M., et al. 2008, ExA, 22, 151

Kellermann, K. I., Fomalont, E. B., Mainieri, V., et al. 2008, ApJS, 179, 71

Komatsu, E., Smith, K. M., Dunkley, J., et al. 2011, ApJS, 192, 18K

Körding, E., Rupen, M., Knigge, C., et al. 2008, Sci, 320, 1318

Kyutoku, K., Ioka, K., \& Shibata, M. 2012, arXiv: 1209.5747

Lazio, J., Bloom, J. S., Bower, G. C., et al. 2009, Astro2010: The Astronomy and Astrophysics Decadal Survey, 2010 (Washington, D.C.: The National Academies Press), 176

Lovell, J. E. J., Rickett, B. J., Macquart, J.-P., et al. 2008, ApJ, 689, 108

Magnelli, B., Elbaz, D., Chary, R. R., et al. 2009, A\&A, 496, 57

Magnelli, B., Elbaz, D., Chary, R. R., et al. 2011, A\&A, 528, 35

Metzger, B. D., \& Berger, E. 2012, ApJ, 746, 48

Miller, N. A., Bonzini, M., Fomalont, E. B., et al. 2013, ApJS, 205, 13

Miller, N. A., Fomalont, E. B., Kellermann, K. I., et al. 2008, ApJS, 179, 114

Murphy, T., Chatterjee, S., Kaplan, D. L., et al. 2012, PASA, 30, e006

Nakar, E., \& Piran, T. 2011, Natur, 478, 82

Nissanke, S., Kasliwal, M., \& Georgieva, A. 2013, ApJ, 767, 124

Norris, R. P., Afonso, J., Appleton, P. N., et al. 2006, AJ, 132, 2409

Ofek, E. O., Breslauer, B., Gal-Yam, A., et al. 2010, ApJ, 711, 517

Ofek, E. O., \& Frail, D. A. 2011, ApJ, 737, 45

Ofek, E. O., Frail, D. A., Breslauer, B., et al. 2011, ApJ, 740, 65

Oosterloo, T., Verheijen, M., \& van Cappellen, W. 2010, ISKAF2010 Science Meeting, arXiv:1007.5141

Padovani, P. 2011, MNRAS, 411, 1547

Perley, R. A., Chandler, C. J., Butler, B. J., \& Wrobel, J. M. 2011, ApJL, 739, L1

Piran, T., Nakar, E., \& Rosswog, S. 2012, MNRAS, 430, 2121

Rix, H.-W., Barden, M., Beckwith, S. V. W., et al. 2004, ApJS, 152, 163

Sadler, E. M., Ricci, R., Ekers, R. D., et al. 2006, MNRAS, 371, 898

Scheers, B. 2011, PhD thesis, Univ. Amsterdam

Schinnerer, E., Smolčić, V., Carilli, C. L., et al. 2007, ApJS, 172, 46

Seymour, N., Dwelly, T., Moss, D., et al. 2008, MNRAS, 386, 1695

Smolčić, V., Schinnerer, E., Scodeggio, M., et al. 2008, ApJS, 177, 14

Soderberg, A. M., Chakraborti, S., Pignata, G., et al. 2010, Natur, 463, 513

Thyagarajan, N., Helfand, D. J., White, R. L., \& Becker, R. H. 2011, ApJ, 742, 49T

Treister, E., Virani, S., Gawiser, E., et al. 2009, ApJ, 693, 1713

Vanzella, E., Cristiani, S., Dickinson, M., et al. 2008, A\&A, 478, 83

Willner, S. P., Ashby, M. L. N., Barmby, P., et al. 2012, ApJ, 756, 72

Wilman, R. J., Jarvis, M. J., Mauch, T., Rawlings, S., \& Hickey, S. 2010, MNRAS, 405, 447

Wolf, C., Meisenheimer, K., Kleinheinrich, M., et al. 2004, A\&A, 421, 913

Zauderer, B. A., Berger, E., Soderberg, A. M., et al. 2011, Natur, 476, 425 